Pacific

Journal of

Mathematics

AN EXTENSION PROCEDURE FOR MANIFOLDS WITH BOUNDARY

JEREMY WONG

Volume $235 \quad$ No. 1 


\title{
AN EXTENSION PROCEDURE FOR MANIFOLDS WITH BOUNDARY
}

\author{
JEREMY WONG
}

\begin{abstract}
This paper introduces an isometric extension procedure for Riemannian manifolds with boundary, which preserves some lower curvature bound and produces a totally geodesic boundary. As immediate applications of this construction, one obtains in particular upper volume bounds, an upper intrinsic diameter bound for the boundary, precompactness, and a homeomorphism finiteness theorem for certain classes of manifolds with boundary, as well as a characterization up to homotopy of Gromov-Hausdorff limits of such a class.
\end{abstract}

\section{Introduction}

For manifolds with boundary, there seems to be a strong connection between the existence of a curvature-controlled extension and uniqueness or finiteness results.

For example, in proving a uniqueness theorem for minimal surfaces, Nitsche [1973] used an extension procedure to extend a minimal surface with boundary a fixed distance beyond its boundary (as a minimal surface). According to a theorem of Lewy [1951], if a minimal surface in $\mathbb{R}^{3}$ (possibly with interior branch points) has a boundary consisting of real analytic boundary curves, then the surface can be extended beyond its boundary as a minimal surface.

Motivated by the Penrose conjecture, Bartnik [1993] considered the extension problem in the class of positive-mass metrics. This problem states, given a bounded Riemannian three-manifold, describe the class of complete three-manifolds satisfying the conditions of the positive mass theorem (in particular, asymptotic flatness with nonnegative scalar curvature) and containing the original three-manifold isometrically. By solving a parabolic PDE, he established a special case of the Penrose conjecture, assuming in particular a foliation of 3-space by metric 2-spheres of positive Gaussian curvature, and boundary data being a minimal surface. The conclusion was that the Schwarzschild metric was distinguished as having the least total mass among all such 3-metrics.

MSC2000: primary 53C20, 53C21; secondary 51K 10.

Keywords: manifold with boundary, extension, Gromov-Hausdorff topology.

The author was supported in part by NSF fellowship. 
Thus, it is important to find general isometric, curvature-controlled extension procedures.

The implicit function theorem and the Cauchy-Kowalevski theorem (in the analytic category) are common tools to extend a manifold with boundary. However, not only do these have a short, if not merely infinitesimal extension range, these procedures are also, unlike the methods of the results mentioned above, insensitive to curvature constraints.

In the context of Riemannian manifolds with nonnegative sectional curvature and locally convex boundary, Kronwith [1979] considered $C^{2}$ extensions preserving nonnegative sectional curvature and locally convex boundary, but his approach relied on power series expansions of the metric tensor and features special to twodimensional surfaces which do not seem to work for dimensions higher than two.

Whitney extension of the metric tensor coefficients is another viable procedure, but to control sectional curvature of such an extension, one must assume a bound on the first two covariant derivatives of the curvature tensor of the original manifold. This is generally regarded as too strong a geometric assumption to make. If one is not interested in isometric extensions, meaning extensions leaving the metric tensor on the original manifold intact, then it is possible to combine the Whitney extension with various smoothing techniques to guarantee that the higher-order covariant derivatives of the curvature tensor are bounded.

Normal extension techniques, such as used in [Kim et al. 2005], do provide any preselected uniform extension range, though so far their use seems largely confined to two-dimensional surfaces. This technique leads to focal points, which explains why a lower bound on the second fundamental form of the boundary must typically be assumed.

Here, we introduce an isometric extension procedure for manifolds with boundary of any dimension; this procedure preserves some lower curvature bound and produces a totally geodesic boundary.

Beginning with any Riemannian manifold with boundary $(M, \partial M)$ one may manufacture a collar, which, when isometrically glued to the boundary, yields an Alexandrov space of curvature bounded below. Outside the gluing locus $\partial M$, the resulting extension $\widetilde{M}$ is $C^{\infty}$. Actually, $\widetilde{M}$ is a $C^{0}$ Riemannian manifold with a $C^{1, \alpha}$ differentiable manifold structure. An important feature is that not only is the curvature of $\widetilde{M}$ bounded from below, but $\widetilde{M}$ can be constructed so as to have a totally geodesic boundary.

This (Alexandrov) extension procedure - so-called since the result is an Alexandrov space - has several applications. Among these, for certain classes of manifolds with boundary, are an upper volume bound, an upper bound to the number of boundary components, an upper intrinsic diameter bound to any boundary component, a dimension estimate for Gromov-Hausdorff limits, precompactness, a 
homeomorphism finiteness result, and a characterization up to homotopy of limits.

Further applications are given in [Alexander et al. $\geq 2008$ ], for topological finiteness of all possible locally convex hypersurfaces in $\mathbb{R}^{n}$ spanning a given codimension-2 smooth submanifold, and in [Wong 2007], for studying collapses of manifolds with boundary.

Now we state the main results of this paper more precisely.

Fix $n \geq 2, K^{-}, \lambda^{ \pm}$, and $d \in \mathbb{R}$. Let $M\left(n, K^{-}, \lambda^{ \pm}, d\right)$ denote the class of $n$ dimensional Riemannian manifolds with boundary with lower sectional curvature bound $K_{M} \geq K^{-}$, two-sided second fundamental form bound $\lambda^{-} \leq I I_{\partial M} \leq \lambda^{+}$, and upper intrinsic diameter bound $d(M) \leq d$. Let $M \in \mathcal{M}\left(n, K^{-}, \lambda^{ \pm}, d\right)$.

Theorem 1.1. (i) $\operatorname{vol}(M) \leq V$ for some $V=V\left(n, K^{-}, \lambda^{ \pm}, d\right)$, a universal positive constant.

(ii) $\operatorname{vol}(\partial M) \leq V$ for some constant $V=V\left(n, K^{-}, \lambda^{ \pm}, d\right)<\infty$.

(iii) The intrinsic diameter of any boundary component of $M$ is uniformly bounded above by $d(\partial M) \leq D\left(n, K^{-}, \lambda^{ \pm}, d\right)$.

(iv) $\partial M$ has no more than c components, where $c=c\left(n, K^{-}, \lambda^{ \pm}, d\right)$ is a finite constant.

Next, the class $\mathcal{M}$ may be endowed with the Gromov-Hausdorff topology.

There is a corollary to the upper volume estimate given above.

Corollary 1.2. If a sequence $M_{i} \in M\left(n, K^{-}, \lambda^{ \pm}, d\right) G H$-converges to a metric space $X$ (necessarily compact and geodesic) then the Hausdorff dimension of $X$ satisfies $\operatorname{dim}_{\mathscr{H}} X \leq n$.

Relative volume comparison does not hold for the class $M\left(n, K^{-}, \lambda^{ \pm}, d\right)$ nor for a more restricted class in which one imposes a lower volume bound to the manifold and an intrinsic injectivity radius bound for the boundary. The ratio of the volume of a ball to the volume of a smaller subball may have no upper bound. Consider for instance a neckpinch, in which two concave parts of the boundary are arbitrarily close to each other. Precompactness does nevertheless hold for these classes, which is remarkable since relative volume comparison (within the class itself) is customarily used to prove it.

Theorem 1.3. $\mathcal{M}\left(n, K^{-}, \lambda^{ \pm}, d\right)$ is precompact in the Gromov-Hausdorff topology.

In other words, any sequence of manifolds with boundary in this class contains a subsequence that converges to a compact metric space.

Theorems 1.1(iv) and 1.3 suggest that the class considered there contains a subclass for which there are only finitely many topological types. Indeed, their number is bounded if a lower volume bound is also imposed: 
Theorem 1.4. $\mathcal{M}\left(n, K^{-}, \lambda^{ \pm}, \mathrm{vol} \geq v>0, d\right)$ contains only finitely many homeomorphism classes.

This is the analogue in the bordered case of the Grove-Petersen-Wu theorem.

The extension procedure also allows one to deduce homotopy structure for limits of certain manifolds with boundary.

Theorem 1.5. Suppose $\left\{M_{i}\right\}$ is a sequence of $n$-dimensional Riemannian manifolds with boundary such that $K_{M_{i}} \geq K^{-},\left|I I_{\partial M_{i}}\right| \leq \lambda, d\left(M_{i}\right) \leq d$, and $M_{i} G H$ converge to a limit space. Then there is a Lipschitz homotopy equivalence

$$
\lim _{\mathrm{GH}} M_{i} \simeq \lim _{\mathrm{GH}} \widetilde{M}_{i},
$$

where $\widetilde{M}_{i}$ are the (Alexandrov) extensions of $M_{i}$, as in Proposition 2.1.

In particular, because $\lim _{\mathrm{GH}} M_{i}$ is homotopic to the locally contractible space $\lim _{\mathrm{GH}} \widetilde{M}_{i}$, it is itself locally contractible and hence admits a universal cover.

The next result is the main aid to constructing the extension.

Theorem 1.6 [Kosovskiu 2002]. Let $M_{1}$ and $M_{2}$ be two smooth Riemannian manifolds with boundary. Suppose their sectional curvatures are bounded below by $K$ and their boundaries are isometric and have respective second-fundamental forms, the sum of which is positive semidefinite. Then the space obtained by isometrically gluing $M_{1}$ to $M_{2}$ along their common boundary is an Alexandrov space of curvature bounded below by $K$.

Another essential element in the construction of the extension is the devising of a collar with the right curvature properties, which will assume the role of $M_{2}$ above.

Lemma 1.7. Suppose $M$ is any manifold with boundary having $K_{M} \geq K^{-}$and $\lambda^{-} \leq I I_{\partial M} \leq \lambda^{+}$. Then for any $t_{0}>0$, there exists an intrinsic metric on $\partial M \times\left[0, t_{0}\right]$ such that $I I_{\partial M \times\{0\}} \geq\left|\min \left\{0, \lambda^{-}\right\}\right|$and $I I_{\partial M \times\left\{t_{0}\right\}} \equiv 0$ and the sectional curvature of $\partial M \times\left[0, t_{0}\right]$ is bounded below by a constant $c\left(K^{-}, \lambda^{ \pm}, t_{0}\right)$.

Letting $C_{M}=\partial M \times\left[0, t_{0}\right]$ denote the collar so produced, the extension $\widetilde{M}$ is defined as

$$
\widetilde{M}=M \bigcup_{\partial M} C_{M} .
$$

The metric on $C_{M}$ is constructed so that $\partial M \times\{0\}$ in $C_{M}$ has a degree of convexity which is at least as great as any possible concavity of $\partial M$ in $M$. The properties of the extension $\widetilde{M}$ are given in more detail in Section 2.1 .

By combining Lemma 1.7 with a similar, but different, gluing technique for Ricci curvature, Theorems 1.1 and 1.3 may be improved.

Theorem 1.8 [Perelman 1997]. Let $M_{1}$ and $M_{2}$ be two $C^{\infty}$ Riemannian manifolds with compact isometric boundaries, each having $\operatorname{ric}\left(M_{i}\right)>r^{-}$for some $r^{-} \in \mathbb{R}$, and 
boundaries satisfying $I I_{\partial M_{1}}+I I_{\partial M_{2}} \geq 0$ at every point on the identified boundary. Then for all $\delta>0$ sufficiently small (depending on $M_{1}$ and $M_{2}$ ) the induced metric on $M_{1} \cup M_{2}$ may be smoothed in a $\delta$-small neighborhood of the gluing locus $\partial M_{i}$ to a $C^{2}$ manifold with $\operatorname{ric}\left(M_{1} \cup M_{2}\right)>r^{-}$.

Originally Theorem 1.8 was sketched in [Perelman 1997] for the case of positive Ricci curvature and positive definite sum of second fundamental forms. The details were provided in [Wang 1997]. It is not hard to check that this generalizes to any lower Ricci curvature bound, as long as the sum condition on the second fundamental forms is satisfied.

Let $\mathcal{M}\left(n, r^{-}, \lambda^{ \pm}, d\right)$ denote the class of $n$-dimensional Riemannian manifolds with boundary with lower Ricci curvature bound $\operatorname{ric}(M) \geq r^{-}$, two-sided second fundamental form bound $\lambda^{-} \leq I_{\partial M} \leq \lambda^{+}$, and upper intrinsic diameter bound $d(M) \leq d$. Let $M \in M\left(n, r^{-}, \lambda^{ \pm}, d\right)$.

Theorem 1.9. (i) $\operatorname{vol}(M) \leq V\left(n, r^{-}, \lambda^{ \pm}, d\right)$.

(ii) $\partial M$ has no more than $c$ components, where $c=c\left(n, r^{-}, \lambda^{ \pm}, d\right)$ is a finite constant.

(iii) The intrinsic diameter of any boundary component of $M$ is uniformly bounded above by $d(\partial M) \leq D\left(n, r^{-}, \lambda^{ \pm}, d\right)$.

(iv) $\mathcal{M}\left(n, r^{-}, \lambda^{ \pm}\right)$is precompact in the pointed Gromov-Hausdorff topology.

This theorem is fairly sharp, in that counterexamples arise if any of the hypotheses, except perhaps the upper bound $\lambda^{+}$, are dropped. The lower bound $\lambda^{-}$(or at least a lower mean curvature bound $H^{-}$put in its place) is necessary for (i), (iii), and (iv) to hold, as examples show. See Figure 1.

For another example, consider removing from the standard unit sphere arbitrarily many small disjoint balls. Thus, the bound $\lambda^{-}$is also necessary for part (ii) of Theorem 1.9.

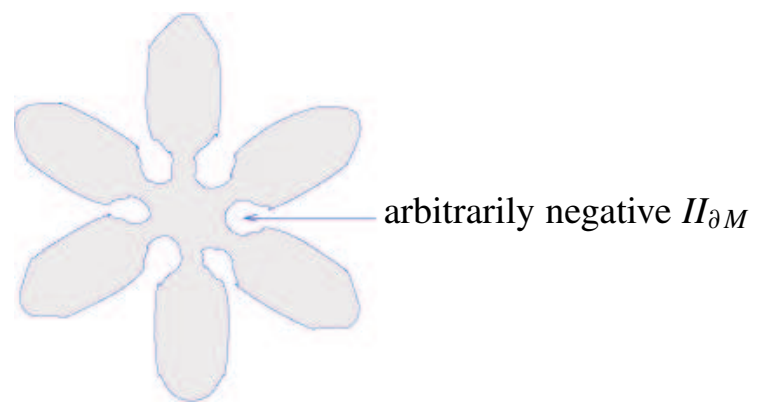

Figure 1. A sunflower with arbitrarily many petals. 
When $n=2$, Lemma 1.7 — and hence Theorems 1.1-1.5 and Theorem 1.9remains true if the upper bound $\lambda^{+}$is dropped.

This paper is organized as follows. After a few additional remarks putting the methods and results into context, Section 2.1 constructs the extension. The main result is Proposition 2.1. Section 2.2 proves Theorems 1.1-1.5 and Theorem 1.9.

\section{Remarks on gluing}

The key assumption of positive semidefiniteness of the sum of the second fundamental forms of the boundaries in the gluing procedure of Kosovskiu [2002] and Perelman [1997] has also been used by Miao [2002]. He obtained a metric of bounded scalar curvature on the union of two manifolds with boundary, assuming that each manifold had bounded scalar curvature and that the boundaries satisfied the sum condition for mean curvature. See [Miao 2002, Proposition 3.1] for details and the proof. The sum condition for mean curvature was proposed in print in [Bartnik 1997]. Recently it has been used to investigate versions of the positive mass theorem [Miao 2002; Shi and Tam 2004].

However, the gluing theorem for Ricci curvature, Theorem 1.8, will not hold in general if the sum condition on the second fundamental forms is replaced by a sum condition on the mean curvatures. See [Wei 1989, page 19] for a counterexample.

One of the earliest references to a sum condition is [Alexandrov 1948], translated in [Aleksandrov and Zalgaller 1967], in which the gluing of several domains with boundary - each cut out of a two-dimensional space of nonnegative curvature (by which is meant either a convex polyhedron, a convex surface, or a $C^{\infty}$ Riemannian manifold) and having sum of turns $\geq 0$ - itself was a space of nonnegative curvature. The notion of turn is a generalization of the integral of geodesic curvature and coincides with it when the boundary is at least $C^{2}$ smooth.

If one is interested in studying a class of manifolds with boundary via the technique of gluing, it is necessary to produce a compatible gluand to attach to a boundary. If this is done smoothly, this is the Cauchy extension problem.

\section{Remarks on [Kodani 1990]}

Theorems 1.3 and 1.4 amend and sharpen certain results of Kodani, whose [1990] paper was one of the earliest to consider convergence of Riemannian manifolds with boundary with respect to the Gromov-Hausdorff topology.

Theorem 1.10 [Kodani 1990]. In $\mathcal{M}\left(n, K^{ \pm}, \lambda^{ \pm}, i_{\partial}, i_{\text {int }}\right)$, the Gromov-Hausdorff and Lipschitz topologies coincide, that is, for every $\delta>0$ there exists an $\epsilon>0$ such that for all $M, N \in \mathcal{M}, d_{G H}(M, N)<\epsilon$ implies $d_{L}(M, N)<\delta$.

Here, the assumed uniform lower bounds on the injectivity radii $i_{\partial}$ and $i_{\text {int }}$ provide a uniform lower volume bound to any $M \in \mathcal{M}$. Refer to Appendix A 
for the definitions of these injectivity radii. Theorem 1.10 is a generalization of Gromov's convergence theorem (see [Gromov 1999, Chapter 8+, Section D] and its references), which asserts that for any sequence of closed $n$-dimensional Riemannian manifolds $M_{i}$ satisfying $\left|K_{M_{i}}\right| \leq K, d\left(M_{i}\right) \leq d$, and $\operatorname{vol}\left(M_{i}\right) \geq v>0$, there is a subsequence that convergences in Lipschitz distance to an $n$-dimensional differentiable manifold with a metric of $C^{1, \alpha}$ Hölder class.

Kodani gave precompactness results for two classes of manifolds with boundary. The first class involved manifolds with locally convex boundary:

Proposition 1.11. $\mathcal{M}\left(n, K^{ \pm}, \lambda^{-}=0, \lambda^{+}, d\right.$, vol $\left.\geq v\right)$ is contained in the class of Theorem 1.10. Furthermore, $M\left(n, K^{ \pm}, \lambda^{-}=0, \lambda^{+}, d\right.$, vol $\left.\geq v\right)$ is precompact in the Gromov-Hausdorff topology.

Kodani also states, but with a circular $\operatorname{proof}^{1}$, the following proposition:

Proposition 1.12. There exist $\lambda_{0}^{-}<0$ and $i_{0}>0$ depending on $n, K^{ \pm}, \lambda^{+}, d$, vol $\geq v$, and $d_{\partial}$, such that $i_{\partial}(M) \geq i_{0}$ and $i_{\text {int }}(M) \geq i_{0}$ is satisfied by any manifold with boundary $M$ in the class $M\left(n, K^{ \pm}, \lambda_{0}^{-}, \lambda^{+}, d\right.$, vol $\left.\geq v, d_{\partial}\right)$, and so $M$ is contained in the class of Theorem 1.10. The class $M\left(n, K^{ \pm}, \lambda_{0}^{-}, \lambda^{+}, d\right.$, vol $\left.\geq v, d_{\partial}\right)$ is precompact in the Gromov-Hausdorff topology.

Here the bound $d_{\partial}$ denotes a fixed upper bound for the sum of the intrinsic diameters of all components of the boundary. In Proposition 1.12, some concavity of the boundary is allowed, but not too much (depending on the other geometric bounds).

By a result of Shikata [1966], if the Lipschitz distance $d_{L}(M, N)$ is sufficiently small for two differentiable spaces $M$ and $N$, then they are diffeomorphic. So Theorem 1.10 implies

Corollary 1.13 [Kodani 1990]. The classes in Propositions 1.11 and 1.12 contain only finitely many diffeomorphism classes. In particular, the number of connected components of the boundary of any manifold in such a class is uniformly bounded above.

The method used to prove Theorem 1.10 consisted of studying inward equidistant parallels of the boundary, relying on the $i_{\partial}$ bound.

\footnotetext{
${ }^{1}$ [Kodani 1990, Proposition 6.2], used to prove Proposition 1.12 above, relies on [Lemma 3.4], which requires the geodesic emanating from a point on the boundary to lie within an $i_{\partial}$ tubular neighborhood of the boundary. It may happen that in [Proposition 6.2] the minimizing $M$-geodesic $\tau_{2}$ (which begins on $\partial M$ ) goes outside such a neighborhood, so that $\tau_{2} \nsubseteq M\left[0, \min \left\{t_{0}, i_{\text {int }}, i_{\partial}\right\}\right]$. In this case, the differential inequality used to derive an upper bound for $l(s):=d\left(\tau_{2}(s), \partial M\right)$ is only valid for certain $0 \leq s \leq s_{1}$, such that $\tau_{2}(s)$ lies within the $i_{\partial}$ neighborhood of $\partial M$. So the upper bound on $l(s)$ holds only if an upper bound on $l(s)$ holds, which is a circular argument invalidating [Proposition 6.2] and thereby the proof of Proposition 1.12 given in [Kodani 1990].
} 
Examples show that Theorem 1.10 is sharp, in that none of the bounds may be dropped. On the contrary, Propositions 1.11 and 1.12 and Corollary 1.13 above are far from optimal, as Theorems 1.3 and 1.4 show. In particular, for precompactness, it is not necessary to assume either an upper bound on the sectional curvature or a lower bound for volume.

\section{Notations and conventions}

Manifolds are assumed to be metrically complete and also, unless we are speaking about the boundary, usually connected. A closed manifold is one that is compact and without boundary.

For an immersion $N \hookrightarrow M$, an inequality of the form $I I \geq \lambda$ signifies that all eigenvalues of the associated quadratic form $S: T N \rightarrow T N$ are $\geq \lambda$. Here we define $I I(X, Y)=g\left(\nabla_{X} v, Y\right)$, where $v$ is the outer normal. By convention, the standard flat disc $D^{2}(r)$ of radius $r$ has $I I=1 / r \geq 0$ and a convex boundary.

If $N$ is a disconnected Riemannian manifold, an inequality of the form $d(N) \leq d$ will usually be interpreted to mean that every path component of $N$ has an upper intrinsic diameter bound $d$. Finally, we adopt these notations:

- $[x y]_{X}$, for a length space $X$, is a minimal geodesic segment from the point $x$ to the point $y$.

- $B(x, r ; X)$ is an open metric ball in $X$ of radius $r$ centered at $x$.

- $d_{X}, d$, or $|\cdot|_{X}$ interchangeably denote the metric distance function of a metric space $X$.

- $\mathcal{M}\left(n, K^{ \pm}, \lambda^{ \pm}, i_{\text {int }}, i_{\partial}, d\right)$ for instance, denotes the class of $n$-dimensional manifolds with boundary $M$ having lower $\left(K^{-}\right)$and upper $\left(K^{+}\right)$interior sectional curvature bounds, lower $\left(\lambda^{-}\right)$and upper $\left(\lambda^{+}\right)$bounds on the second fundamental form, some (unspecified) uniform positive lower bounds to $i_{\text {int }}(M)$ and $i_{\partial}(M)$, and an upper diameter bound $d$.

\section{2. (Alexandrov) extension}

\subsection{Construction.}

Proof of Lemma 1.7. Let $\bar{\lambda}=\min \left\{0, \lambda^{-}\right\}$. Fix some $t_{0}>0$ and $0<\epsilon<1$. For some $K=K\left(\lambda, \epsilon, t_{0}\right) \in \mathbb{R}$, there exists a $C^{\infty}$ monotone nonincreasing function $\phi(t)$ defined on $\left[0, t_{0}\right]$ satisfying (see Figure 2 )

$$
\begin{aligned}
& \phi^{\prime \prime}+K \phi \leq 0, \quad \phi(0)=1, \quad \phi\left(t_{0}\right)=\epsilon, \\
& -\infty<\phi^{\prime}(0) \leq \bar{\lambda}, \quad \phi^{\prime}\left(t_{0}\right)=0 .
\end{aligned}
$$

Consider the warped-product metric on $\partial M \times\left[0, t_{0}\right]$ given by $g_{1}(x, t)=d t^{2}+$ $\phi(t)^{2} g_{\partial M}(x)$. Let $X$ and $Y$ be $g_{1}$-orthonormal tangent vectors to $\partial M \times\left[0, t_{0}\right]$ at 


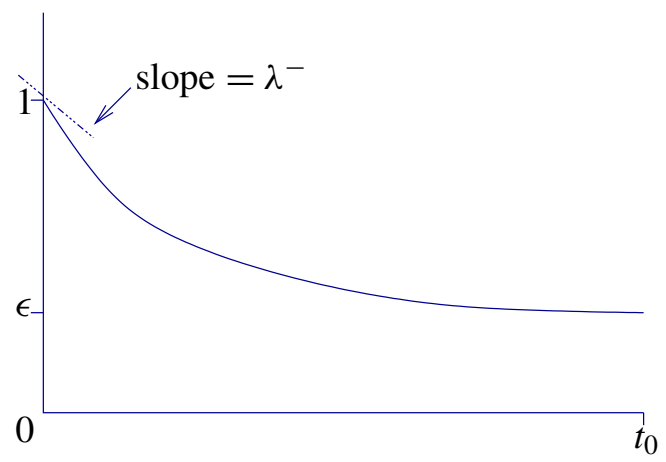

Figure 2. Warping function $\phi$.

the point $(x, t)$ that are vertical with respect to the Riemannian submersion that projects $\left(\partial M \times\left[0, t_{0}\right], g_{1}\right)$ onto $\left[0, t_{0}\right]$. Let $T$ denote a radial (that is, a horizontal) unit tangent vector to $\partial M \times\left[0, t_{0}\right]$ at $(x, t)$. From the O'Neill formula for a Riemannian submersion, $g_{1}$ by construction has tangential sectional curvatures and radial curvatures

$$
K_{1}(X, Y)=\frac{K_{\partial M}(X, Y)-\left|\phi^{\prime}(t)\right|^{2}}{\phi^{2}(t)} \quad \text { and } \quad K_{1}(X, T)=-\frac{\phi^{\prime \prime}(t)}{\phi(t)} \geq K \in \mathbb{R},
$$

respectively. If $X_{i}$ and $X_{j}$ are any unit vertical tangent vectors,

$$
I I_{X_{i} \wedge X_{j}}(x, 0)=-\frac{\phi^{\prime}(0)}{\phi(0)} \geq|\bar{\lambda}| \quad \text { and } \quad I I_{X_{i} \wedge X_{j}}\left(x, t_{0}\right)=-\frac{\phi^{\prime}\left(t_{0}\right)}{\phi\left(t_{0}\right)}=0,
$$

so that the boundary is locally convex as stated.

In Lemma 1.7, $t_{0}$ and $\epsilon$ are independent free parameters which may be chosen according to one's purpose. The optimal (that is, the greatest) lower bound $K_{0}$ achievable for some $\phi$ satisfying the above requirements decreases to $-\infty$ as $t_{0}$ decreases to 0 (with $\epsilon$ fixed). It also decreases to $-\infty$ as $\epsilon$ tends to 1 (with $t_{0}$ fixed), provided $\lambda^{-}<0$ is fixed too.

An explicit warping function $\phi$. Here is an explicit construction of a warping function $\phi$ satisfying the condition of the lemma.

Assume that $0<\epsilon<1,0<t_{0}$, and $\bar{\lambda} \leq 0$. It may be further assumed that $\bar{\lambda}<0$, since otherwise $\lambda^{-} \geq 0$, and then the boundary, being locally convex, would not require an extension (in this case one could just take $\phi \equiv 1$ ).

For $0 \leq t<t_{0}$, define

$$
\phi(t)=(1-\epsilon) \exp \left(\frac{\bar{\lambda} t_{0}^{2}}{1-\epsilon}\left(\frac{1}{t_{0}-t}-\frac{1}{t_{0}}\right)\right)+\epsilon .
$$


Then

$$
\phi^{\prime}(t)=\frac{\bar{\lambda} t_{0}^{2}}{\left(t_{0}-t\right)^{2}} \exp \left(\frac{\bar{\lambda} t_{0}^{2}}{1-\epsilon}\left(\frac{1}{t_{0}-t}-\frac{1}{t_{0}}\right)\right) .
$$

Extend $\phi$ to be defined on $\left[0, t_{0}\right]$ by requiring continuity of $\phi$ and all its derivatives, that is, $\phi\left(t_{0}\right):=\lim _{t \uparrow t_{0}} \phi(t), \phi^{\prime}\left(t_{0}\right):=\lim _{t \uparrow t_{0}} \phi^{\prime}(t)$, and so on.

Then

$$
\begin{aligned}
\phi(0) & =1, & \phi\left(t_{0}\right) & =\lim _{t \uparrow t_{0}} \phi(t)=(1-\epsilon) \cdot 0+\epsilon=\epsilon, \\
\phi^{\prime}(0) & =\bar{\lambda}, & \phi^{\prime}\left(t_{0}\right) & =\lim _{t \uparrow t_{0}} \phi^{\prime}(t)=0,
\end{aligned}
$$

and obviously $\phi^{\prime}(t) \leq 0$ for all $0 \leq t \leq t_{0}$ (so $\phi$ is nonincreasing) since $\bar{\lambda} \leq 0$. Now

$$
\phi^{\prime \prime}(t)=\left(2 \frac{\bar{\lambda} t_{0}^{2}}{\left(t_{0}-t\right)^{3}}+\frac{(\bar{\lambda})^{2} t_{0}^{4}}{\left(t_{0}-t\right)^{4}(1-\epsilon)}\right) \exp \left(\frac{\bar{\lambda} t_{0}^{2}}{1-\epsilon}\left(\frac{1}{t_{0}-t}-\frac{1}{t_{0}}\right)\right)
$$

has four possible critical points $0, t_{0}$, and the two points

$$
t_{3 \pm}=t_{0}+\frac{1}{6} \frac{(3 \pm \sqrt{3}) \bar{\lambda} t_{0}^{2}}{1-\epsilon},
$$

with corresponding values $\phi^{\prime \prime}(0)=2 \bar{\lambda} / t_{0}+\bar{\lambda}^{2} /(1-\epsilon), \phi^{\prime \prime}\left(t_{0}\right)=\lim _{t \uparrow t_{0}} \phi^{\prime \prime}(t)=0$, and

$$
\phi^{\prime \prime}\left(t_{3 \pm}\right)=\mp 432 \frac{\sqrt{3}(1-\epsilon)^{3}}{\bar{\lambda}^{2} t_{0}^{4}(3 \pm \sqrt{3})^{4}} \exp \left(-\frac{6}{3 \pm \sqrt{3}}-\frac{\bar{\lambda} t_{0}}{1-\epsilon}\right) .
$$

But $t_{3 \pm} \in\left[0, t_{0}\right]$ if and only if

$$
\frac{|\bar{\lambda}| t_{0}}{1-\epsilon} \leq \frac{6}{3 \pm \sqrt{3}}
$$

Observe that $1 \geq \phi(t) \geq \epsilon>0$ for all $0 \leq t \leq t_{0}$. If $\phi^{\prime \prime}(t)<0$ then $-\phi^{\prime \prime}(t) / \phi(t) \geq 0$. If $\phi^{\prime \prime}(t)>0$ then

$$
\begin{aligned}
-\frac{\phi^{\prime \prime}(t)}{\phi(t)} \geq-\frac{1}{\epsilon} \max \phi^{\prime \prime}(t) & =-\frac{1}{\epsilon} \max \left\{\phi^{\prime \prime}(0), \phi^{\prime \prime}\left(t_{0}\right), \phi^{\prime \prime}\left(t_{3 \pm}\right)\right\} \\
& =-\frac{1}{\epsilon} \max \left\{0,\left(2 \frac{\bar{\lambda}}{t_{0}}+\frac{\bar{\lambda}^{2}}{1-\epsilon}\right), \phi^{\prime \prime}\left(t_{3-}\right)\right\}
\end{aligned}
$$

provided that (2-3) holds for both cases. (Even if they do not, the bound here is still valid, though not as sharp.) Therefore in any case, the radial curvatures are bounded below according to (2-4).

It remains to bound the tangential curvatures from below.

The derivative $\phi^{\prime}(t)$ itself has at most three possible critical points $0, t_{0}$, and $t_{4}=t_{0}+\bar{\lambda} t_{0}^{2} /(2(1-\epsilon))$ in $\left[0, t_{0}\right]$, with corresponding values $\phi^{\prime}(0)=\bar{\lambda}, \phi^{\prime}\left(t_{0}\right)=0$, 
and

$$
\phi^{\prime}\left(t_{4}\right)=4 \frac{(1-\epsilon)^{2}}{\bar{\lambda} t_{0}^{2}} \exp \left(-2-\frac{\bar{\lambda} t_{0}}{1-\epsilon}\right)
$$

But note that $t_{4} \in\left[0, t_{0}\right]$ if and only if $|\bar{\lambda}| t_{0} /(1-\epsilon) \leq 2$.

Now note

$$
\begin{array}{ll}
K_{\partial M}^{-} / \phi\left(t^{2}\right) \geq 0 & \text { if } K_{\partial M}^{-} \geq 0, \\
K_{\partial M}^{-} / \phi\left(t^{2}\right) \geq K_{\partial M}^{-} / \epsilon^{2} & \text { if } K_{\partial M}^{-} \leq 0 .
\end{array}
$$

Therefore in any case, the tangential sectional curvatures are bounded below by

$$
\begin{aligned}
& \frac{1}{\phi^{2}(t)}\left(K_{\partial M}^{-}-\left|\phi^{\prime}(t)\right|^{2}\right) \geq \min \left\{0, K_{\partial M}^{-} / \epsilon^{2}\right\}-\max \left|\phi^{\prime}(t)\right|^{2} / \min \phi^{2}(t) \\
& \geq \frac{1}{\epsilon^{2}}\left(\min \left\{0, K^{-}-\left(\max \left\{\left|\lambda^{-}\right|,\left|\lambda^{+}\right|\right\}\right)^{2}\right\}-\max \left\{|\bar{\lambda}|^{2},(\text { value of }(2-5))^{2}\right\}\right) .
\end{aligned}
$$

If in an orthonormal frame the sectional curvatures are bounded (from below) on all frame two-planes, then the sectional curvatures are bounded (from below) on arbitrary two-planes. So (2-4) and (2-6) together prove that the sectional curvatures of $\left(\partial M \times\left[0, t_{0}\right], g_{1}\right)$ are bounded below by a constant $c\left(K^{-}, \lambda^{ \pm}, t_{0}\right)$, as stated in Lemma 1.7 .

Proposition 2.1 (Construction of extension). Fix $n \geq 2$ and $K^{-}, \lambda^{ \pm} \in \mathbb{R}$. For any $M \in \mathcal{M}\left(n, K^{-}, \lambda^{ \pm}\right) \equiv\left\{M\right.$ Riemannian n-manifold: $\left.K^{-} \leq K_{M}, \lambda^{-} \leq I I_{\partial M} \leq \lambda^{+}\right\}$, there exists an isometric, uniform extension $\widetilde{M}$ of $M$ that is an Alexandrov space of curvature bounded below by a constant $k=k\left(K^{-}, \lambda^{ \pm}\right)$. The extension is uniform in that the distance in $\widetilde{M}$ between $\partial M$ and $\partial \widetilde{M}$ is no smaller than a constant which may be chosen arbitrarily.

Proof. Choose some $t_{0}>0$ and $1>\epsilon>0$. Construct $\widetilde{M}$ as follows. Let $C_{M}=$ $\partial M \times\left[0, t_{0}\right]$, and equip it with the metric $g_{1}$ of Lemma 1.7. Let $\widetilde{M}:=M \cup C_{M}$ be the isometric gluing of $C_{M}$ to $M$ along their isometric boundaries. Now let $K_{C}^{-}=K_{C}^{-}\left(K^{-}, \lambda^{ \pm}\right)$denote the lower sectional curvature bound of the collar produced in the lemma. Then by Theorem 1.6, $\widetilde{M}$ is an Alexandrov space of curvature bounded below by $\min \left\{K^{-}, K_{C}^{-}\right\}$. The last claim follows by construction of the metric in Lemma 1.7, since geodesics emanating orthogonally to $\partial \widetilde{M}$ minimize the distance to $\partial \widetilde{M}$ at least as long as they remain in $C_{M}$.

We remark that when $\operatorname{dim} M=2$ the upper bound $\lambda^{+}$is not needed in Lemma 1.7 nor in Proposition 2.1 and its corollaries, since in this situation there are no tangential two-planes on which to speak of curvature.

Note also that under only the hypotheses of Proposition 2.1, neither $M$ nor $C_{M}$ need be a locally convex subset of $\widetilde{M}$. For instance, to see that $C_{M}$ need not be locally convex in $\widetilde{M}$, take $M$ to be the result of cutting lengthwise (through the 
apex) a rounded-off cone with small cone angle, so that the resulting boundary of $M$ near the apex is totally geodesic, but elsewhere has some concavity.

Properties of $\widetilde{\boldsymbol{M}}$. By [Kosovskiu 2002], a gluing as in Theorem 1.6, such as the $\widetilde{M}$ constructed in Proposition 2.1, is realized as the limit of manifolds with metric tensors of class $W_{\text {loc }}^{2, \infty}$ that have lower curvature bounds approaching that of $\widetilde{M}$. These in turn are constructed using Sobolev averaging of the metrics in a neighborhood of the gluing locus. As a consequence, $\widetilde{M}$ is a $C^{1, \alpha}$ differentiable manifold with $C^{0}$ Riemannian structure and is almost everywhere $C^{\infty}$.

For future reference, we record these additional properties of the extension $\widetilde{M}$ :

(i) $i_{\partial}(\tilde{M}) \geq t_{0}$.

(ii) $d(\widetilde{M}) \leq d(M)+2 t_{0}$.

(iii) $|x y|_{M} \leq \frac{1}{\epsilon}|x y|_{\widetilde{M}}$ for all $x, y \in M$. In particular, $d(M) \leq \frac{1}{\epsilon} d(\widetilde{M})$.

(iv) $\partial M=\frac{1}{\epsilon} \partial \widetilde{M}$. In particular, $d(\partial M)=\frac{1}{\epsilon} d(\partial \widetilde{M})$.

Properties (i) and (iv) are clear from construction. Define a map $\pi: \widetilde{M} \rightarrow M$ by

$$
\pi(x)= \begin{cases}x & \text { if } x \in M, \\ \text { orthogonal projection of } x \text { onto } \partial C_{M}=\partial M & \text { if } x \in C_{M} .\end{cases}
$$

Let $x, y \in \widetilde{M}$. Then

$$
\begin{array}{rlr}
|x y|_{\widetilde{M}} & \leq|x \pi(x)|_{\widetilde{M}}+|\pi(x) \pi(y)|_{\widetilde{M}}+|\pi(y) y|_{\widetilde{M}} & \text { by the triangle inequality } \\
& \leq t_{0}+|\pi(x) \pi(y)|_{M}+t_{0} & \text { since } M \subset \widetilde{M} \\
& \leq d(M)+2 t_{0},
\end{array}
$$

which proves (ii). Property (iii) will be proved in the course of Lemma 2.2.

The Lipschitz continuity (as well as the Lipschitz constant) of the projection map $\pi$ from $\widetilde{M}$ to $M$ will be important in later applications.

Lemma 2.2. Let $(M, \partial M)$ be fixed. Consider the projection map $\pi: \widetilde{M} \rightarrow M$ defined above. Then for all $x, y \in \widetilde{M}$, we have $|\pi(x) \pi(y)|_{M} \leq \frac{1}{\epsilon}|x y| \widetilde{M}$.

Proof. Let $\gamma:=[x y]_{\widetilde{M}}$ be a minimal $\widetilde{M}$-geodesic from $x$ to $y$ parametrized on $[0,1]$, and consider an arbitrary partition $0 \leq t_{1} \leq \ldots \leq t_{N} \leq 1$ of $[0,1]$. Conceivably, $\gamma$ may cross $\partial M$ infinitely many times.

If $\left[\gamma\left(t_{i}\right) \gamma\left(t_{i+1}\right)\right]_{\widetilde{M}} \subset M$ then

$$
\left|\pi\left(\gamma\left(t_{i}\right)\right) \pi\left(\gamma\left(t_{i+1}\right)\right)\right|_{\widetilde{M}}=\left|\gamma\left(t_{i}\right) \gamma\left(t_{i+1}\right)\right|_{\widetilde{M}}
$$


If $\left[\gamma\left(t_{i}\right) \gamma\left(t_{i+1}\right)\right]_{\widetilde{M}} \subset C_{M}$ then

$$
\begin{aligned}
\left|\pi\left(\gamma\left(t_{i}\right)\right) \pi\left(\gamma\left(t_{i+1}\right)\right)\right|_{\widetilde{M}} & \leq\left|\pi\left[\gamma\left(t_{i}\right) \gamma\left(t_{i+1}\right)\right]_{\widetilde{M}}\right|_{M} \\
& =\left|\pi\left[\gamma\left(t_{i}\right) \gamma\left(t_{i+1}\right)\right]_{C_{M}}\right|_{M} \\
& =\left|\pi\left[\gamma\left(t_{i}\right) \gamma\left(t_{i+1}\right)\right]_{C_{M}}\right|_{\partial C_{M}} \\
& \leq \frac{1}{\epsilon}\left|\gamma\left(t_{i}\right) \gamma\left(t_{i+1}\right)\right|_{C_{M}}=\frac{1}{\epsilon}\left|\gamma\left(t_{i}\right) \gamma\left(t_{i+1}\right)\right|_{\widetilde{M}} .
\end{aligned}
$$

Otherwise $\left[\gamma\left(t_{i}\right) \gamma\left(t_{i+1}\right)\right]_{\widetilde{M}}$ belongs neither to $M$ nor $C_{M}$. In this case, when $\gamma\left(t_{i}\right) \in C_{M}$, let $t_{i}^{*} \in \partial M$ be such that $\left[\gamma\left(t_{i}\right) \gamma\left(t_{i}^{*}\right)\right]_{\widetilde{M}} \subset C_{M}$; otherwise set $t_{i}^{*}:=t_{i}$. Similarly, if $\gamma\left(t_{i+1}\right) \in C_{M}$, let $t_{i}^{* *} \in \partial M$ be such that $\left[\gamma\left(t_{i}^{* *}\right) \gamma\left(t_{i+1}\right)\right]_{\widetilde{M}} \subset C_{M}$; otherwise set $t_{i}^{* *}:=t_{i+1}$.

Note that $\pi\left(\gamma\left(t_{i}^{*}\right)\right)=\gamma\left(t_{i}^{*}\right)$ and $\pi\left(\gamma\left(t_{i}^{* *}\right)\right)=\gamma\left(t_{i}^{* *}\right)$, so that

$$
\left|\pi\left(\gamma\left(t_{i}^{*}\right)\right) \pi\left(\gamma\left(t_{i}^{* *}\right)\right)\right|_{\widetilde{M}}=\left|\gamma\left(t_{i}^{*}\right) \gamma\left(t_{i}^{* *}\right)\right|_{\widetilde{M}},
$$

just as in (2-8). Then

$$
\begin{aligned}
\mid \pi\left(\gamma\left(t_{i}\right)\right) & \left.\pi\left(\gamma\left(t_{i+1}\right)\right)\right|_{\widetilde{M}} \\
& \leq\left|\pi\left(\gamma\left(t_{i}\right)\right) \pi\left(\gamma\left(t_{i}^{*}\right)\right)\right|_{\widetilde{M}}+\left|\pi\left(\gamma\left(t_{i}^{*}\right)\right) \pi\left(\gamma\left(t_{i}^{* *}\right)\right)\right|_{\widetilde{M}}+\left|\pi\left(\gamma\left(t_{i}^{* *}\right)\right) \pi\left(\gamma\left(t_{i+1}\right)\right)\right|_{\widetilde{M}} \\
& \leq \frac{1}{\epsilon}\left|\gamma\left(t_{i}\right) \gamma\left(t_{i}^{*}\right)\right|_{\widetilde{M}}+\left|\gamma\left(t_{i}^{*}\right) \gamma\left(t_{i}^{* *}\right)\right|_{\widetilde{M}}+\frac{1}{\epsilon}\left|\gamma\left(t_{i}^{* *}\right) \gamma\left(t_{i+1}\right)\right|_{\widetilde{M}} \\
& \leq \frac{1}{\epsilon}\left(\left|\gamma\left(t_{i}\right) \gamma\left(t_{i}^{*}\right)\right|_{\widetilde{M}}+\left|\gamma\left(t_{i}^{*}\right) \gamma\left(t_{i}^{* *}\right)\right|_{\widetilde{M}}+\left|\gamma\left(t_{i}^{* *}\right) \gamma\left(t_{i+1}\right)\right|_{\widetilde{M}}\right) \\
& =\frac{1}{\epsilon}\left|\gamma\left(t_{i}\right) \gamma\left(t_{i+1}\right)\right|_{\widetilde{M}} .
\end{aligned}
$$

where the first inequality uses the triangle inequality, the second uses (2-9) and (2-10), and the third uses $\epsilon \leq 1$. Hence, noting that $\pi[x y]_{\widetilde{M}}$ is a path in $M$ from $\pi(x)$ to $\pi(y)$, we have

$$
\begin{aligned}
|\pi(x) \pi(y)|_{M} \leq|\pi[x y] \widetilde{M}|_{M} & =\limsup \sum_{i=1}^{N-1}\left|\pi\left(\gamma\left(t_{i}\right)\right) \pi\left(\gamma\left(t_{i+1}\right)\right)\right|_{M} \\
& =\limsup \sum_{i=1}^{N-1}\left|\pi\left(\gamma\left(t_{i}\right)\right) \pi\left(\gamma\left(t_{i+1}\right)\right)\right|_{\widetilde{M}} \\
& \leq \limsup \sum_{i=1}^{N-1} \frac{1}{\epsilon}\left|\gamma\left(t_{i}\right) \gamma\left(t_{i+1}\right)\right|_{\widetilde{M}}=\frac{1}{\epsilon}|x y| \widetilde{M},
\end{aligned}
$$

where the lim sup is taken over all partitions $\left(t_{1}, \ldots, t_{N}\right)$ of $[0,1]$ and over all $N$. Here the inequality uses (2-8), (2-9), and the previous estimate.

2.2. Corollaries of the construction. We will now prove Theorems $1.1-1.5$ and Theorem 1.9. For the rest of this section, a single choice of warping function $\phi$, as in the construction of the (Alexandrov) extension, will be fixed. 
Proof of Theorem 1.1(i). From the coarea formula, valid for Alexandrov spaces,

$$
\operatorname{vol}(\widetilde{M})=\int_{0}^{d(\widetilde{M})} \operatorname{vol}_{n-1}(S(x, r ; \widetilde{M})) d r
$$

for any $x \in \tilde{M}$, where $\operatorname{vol}_{n-1}$ stands for the $(n-1)$-dimensional Hausdorff measure and $S(x, r ; \widetilde{M})$ denotes the metric distance sphere of radius $r$ about $x$ in $\widetilde{M}$. In particular, if $S(x, r ; \widetilde{M})=\varnothing$ then its measure is assigned the value zero. Note that $S(x, r ; \widetilde{M}) \cap M=\varnothing$ if $x$ is chosen to lie in $M$ and $r>d(M)$. Hence (for example, [Burago et al. 2001, Theorem 10.6.8]),

$$
\begin{aligned}
\operatorname{vol}(M) & =\int_{0}^{d(M)} \operatorname{vol}_{n-1}(S(x, r ; \tilde{M}) \cap M) d r \\
& \leq \int_{0}^{d(M)} \operatorname{vol}_{n-1}(S(x, r ; \widetilde{M})) d r \\
& \leq \operatorname{vol}\left(S^{n-1}(1)\right) \int_{0}^{d(M)} \operatorname{sn}_{\min \left\{K^{-}, K_{C}^{-}\right\}}^{n-1}(r) d r
\end{aligned}
$$

where

$$
\operatorname{sn}_{k}(r):= \begin{cases}\sin (\sqrt{k} r) / \sqrt{k} & k>0, \\ r & k=0, \\ \sinh (\sqrt{|k| r}) / \sqrt{|k|} & k<0\end{cases}
$$

is the generalized sine function; so the volume is bounded above by a constant $V\left(n, K^{-}, \lambda^{ \pm}, d\right)$.

Proof of Theorem 1.1(ii). Since $C_{M} \subset \widetilde{M}, \operatorname{vol}\left(C_{M}\right) \leq \operatorname{vol}(\widetilde{M}) \leq V\left(n, K^{-}, \lambda^{ \pm}, d\right)$, where the last $V$ is obtained like in Theorem 1.1(i). Since $C_{M}=\partial M \times_{\phi}\left[0, t_{0}\right]$ and $\partial M \times_{\phi}\left\{t_{0}\right\}=\epsilon \cdot\left(\partial M \times_{\phi}\{0\}\right)$,

$$
\operatorname{vol}\left(C_{M}\right) \geq \operatorname{vol}\left(\partial M \times_{\phi}\left\{t_{0}\right\}\right) \cdot t_{0}=\epsilon^{n-1} \cdot \operatorname{vol}\left(\partial M \times_{\phi}\{0\}\right) \cdot t_{0} .
$$

Combining these inequalities yields

$$
\operatorname{vol}(\partial M)=\operatorname{vol}\left(\partial M \times_{\phi}\{0\}\right) \leq \frac{V\left(n, K^{-}, \lambda^{ \pm}, d\right)}{t_{0} \cdot \epsilon^{n-1}} .
$$

Remarks on volume. First, even in dimension 2, Theorem 1.1(i) (not requiring $\lambda^{+}$) is apparently inaccessible by the Gauss-Bonnet formula, isoperimetric inequalities, and the Heintze-Karcher approach of exponentiating the normal bundle of the boundary, which would work if in addition one had an upper volume bound of the boundary itself.

Second, by replacing $d$ with $r$ in Theorem 1.1(i), one also gets a bound for the volume of a ball $B(x, r)$ in any $M \in M\left(n, K^{-}, \lambda^{ \pm}, d\right)$. Neither this nor Theorem 1.1(i) itself is sharp in general. It is sometimes more efficient to add a cap or cone to the boundary. 
In contrast to the closed case, there is no volume rigidity for manifolds with sectional curvature and second fundamental form bounds, unless special restrictions are placed on these values, for example, by assuming the boundary is locally convex. In other words, even though it is possible to find a model space for which

$$
\operatorname{vol}(B(x, r ; M)) \leq \operatorname{vol}(B(\bar{x}, r ; \text { model space }))
$$

holds for every $M \in \mathcal{M}\left(n, K^{-}, \lambda^{ \pm}, d\right)$ and all $x \in M$ and $0<r \leq d$ (we accomplished this above), it will not be possible in general to assert that the ball is isometric to the model space ball when the volume is maximal.

This lack of volume rigidity persists even for small metric balls in the manifold (in particular, those that intersect the boundary) and is related to the observation that there appears to be no universal model space or single class of model spaces. An example may be constructed by considering the manifolds obtained by removing from the unit sphere $S^{n}(1)$ three disjoint metric balls of the same radius but of various configurations.

Proof of Corollary 1.2. For each $M_{i}$, construct $\widetilde{M}_{i}$ using a fixed warping function independent of $i$. By precompactness, the $\widetilde{M}_{i}$ have a Gromov-Hausdorff limit $Y$.

By Lemma 2.2 the maps $\pi_{i}: \widetilde{M}_{i} \rightarrow M_{i}$ are surjective and uniformly Lipschitz. By Proposition B.1, there is a Lipschitz surjection from $Y$ to $X$. Lipschitz maps do not raise Hausdorff dimension, and so $\operatorname{dim}_{\mathscr{H}} X \leq \operatorname{dim}_{\mathcal{H}} Y \leq n$.

We note as an aside that another proof may be obtained from the volume estimate of Theorem 1.1(i). Briefly stated, it suffices to show that the $n$-dimensional Hausdorff measure $\operatorname{vol}_{n}(X)$ of $X$ is finite. Let cov denote the covering function, that is, $\operatorname{cov}(X, r)$ is the minimal number of balls of radius $r$ needed to cover $X$. First note that cov is almost-continuous with respect to GH-convergence, in that if $d_{\mathrm{GH}}\left(M_{i}, X\right) \leq \eta_{i} \rightarrow 0$, then $\operatorname{cov}(X, r) \leq \operatorname{cov}\left(M_{i}, r-\eta_{i}\right)$.

Then, setting $\omega_{n-1}:=\operatorname{vol}\left(S^{n-1}(1)\right)$,

$$
\begin{array}{rlr}
\operatorname{vol}_{n}(X) & :=\lim _{r \downarrow 0} \frac{\omega_{n-1}}{n} r^{n} \operatorname{cov}(X, r) & \text { by definition } \\
& \leq \lim _{r \downarrow 0} \limsup _{i \rightarrow \infty} \frac{\omega_{n-1}}{n} r^{n} \operatorname{cov}\left(M_{i}, r-\eta_{i}\right) & \\
& \leq \lim _{r \downarrow 0} \limsup _{i \rightarrow \infty} \frac{\omega_{n-1}}{n} r^{n} \operatorname{cov}\left(M_{i}, r\right) & \\
& =\limsup _{i \rightarrow \infty} \lim _{r \downarrow 0} \frac{\omega_{n-1}}{n} r^{n} \operatorname{cov}\left(M_{i}, r\right) & =\limsup _{i \rightarrow \infty} \operatorname{vol}_{n}\left(M_{i}\right) \\
& & \leq V\left(n, K^{-}, \lambda^{ \pm}, d\right)<\infty,
\end{array}
$$

where the final " $\leq$ " is by Theorem 1.1(i).

Proof of Theorem 1.1(iii). For any metric space $X$ and radius $r \leq R$, define the capacity $\operatorname{cap}(r, R ; X)$ of $X$ to be the maximum number of disjoint balls of radius $r$ in $X$ that can be packed in a ball of radius $R$. 
Recall that any $M \in \mathcal{M}$ admits an extension $\widetilde{M}$ with $i_{\partial}(\widetilde{M}) \geq t_{0}$. Also, $\partial \widetilde{M}=$ $\epsilon \partial M$, where $\epsilon$ is the fixed parameter given in the extension procedure. For any $0<r \leq R$, it is obvious that

$$
\operatorname{cap}(r, R ; \partial M)=\operatorname{cap}(\epsilon r, \epsilon R ; \epsilon \partial M)=\operatorname{cap}(\epsilon r, \epsilon R ; \partial \widetilde{M})
$$

since the former amounts to a relabeling of the units of distance.

Let $\exp ^{\perp}$ denote the normal exponential map of the boundary $\partial \widetilde{M}$. Observe that if $B_{1}\left(x_{1}, \epsilon r ; \partial \widetilde{M}\right)$ and $B_{2}\left(x_{2}, \epsilon r ; \partial \widetilde{M}\right)$ are any two disjoint balls in $\partial \widetilde{M}$ and $\epsilon r \leq t_{0} / 2$, then there exist disjoint balls $B(\epsilon r ; \widetilde{M})$ and $B(\epsilon r ; \widetilde{M})$ in the cylindrical regions $\left\{\exp _{t}^{\perp}\left(B_{1}\right): 0 \leq t \leq t_{0}\right\}$ and $\left\{\exp _{t}^{\perp}\left(B_{2}\right): 0 \leq t \leq t_{0}\right\}$ of $\widetilde{M}$, respectively, with radii commensurately bounded below. A similar statement holds for a disjoint collection of balls. Thus if $\epsilon r \leq t_{0} / 2$,

$$
\operatorname{cap}(\epsilon r, \epsilon R ; \partial \widetilde{M}) \leq \operatorname{cap}(\epsilon r, d(\widetilde{M}) ; \widetilde{M}) .
$$

But since curv $\widetilde{M} \geq k=k\left(K^{-}, \lambda^{ \pm}\right)$, relative volume comparison in $\widetilde{M}$ implies

$$
\begin{aligned}
\operatorname{cap}(\epsilon r, d(\widetilde{M}) ; \widetilde{M}) & \leq \frac{\operatorname{vol}(B(d(\widetilde{M}) ; \widetilde{M}))}{\operatorname{vol}(B(\epsilon r ; \widetilde{M}))} \\
& \leq \frac{\operatorname{vol}\left(B\left(d+2 t_{0} ; \widetilde{M}\right)\right)}{\operatorname{vol}(B(\epsilon r ; \widetilde{M}))} \\
& \leq \frac{\operatorname{vol}\left(B\left(d+2 t_{0} ; M_{k}^{n}\right)\right)}{\operatorname{vol}\left(B\left(\epsilon r ; M_{k}^{n}\right)\right)} \leq N_{n, k, d}(\epsilon r),
\end{aligned}
$$

where $M_{k}^{n}$ denotes the standard $n$-dimensional, simply-connected model space of constant curvature $k$, and where the quantity $N_{n, k, d}(\epsilon r)$ is independent of $\widetilde{M}$.

On the other hand, let $\gamma:[0, R] \rightarrow \partial M$ be a diametral minimal segment in $\partial M$ parametrized with unit speed so that $R=d(\partial M)$. Suppose $r \leq R$. By the minimality of $\gamma$, the open metric balls $B(\gamma(j 2 r), r ; \partial M)$ for $j=0,1, \ldots,\lfloor R / 2 r\rfloor$ must be disjoint.

Therefore $R / 2 r \leq \operatorname{cap}(r, R ; \partial M)$. Combining this with (2-11)-(2-13) yields

$$
R \leq 2 r N_{n, k, d}(\epsilon r) .
$$

In particular, setting $r=t_{0} / 2 \epsilon$ gives

$$
d(\partial M) \leq \max \left\{\frac{t_{0}}{2 \epsilon}, \frac{t_{0}}{\epsilon} \frac{\int_{0}^{d+2 t_{0}} \mathrm{sn}_{k}^{n-1}(t) d t}{\int_{0}^{t_{0} / 2} \operatorname{sn}_{k}^{n-1}(t) d t}\right\},
$$

and the latter term yields the upper bound.

An exponential dependence of the diameter of the boundary upon the diameter of the manifold itself is necessary in general, as the example of a ball in hyperbolic space shows. One expects that a sharper dimension-free upper bound to the 
intrinsic diameter of the boundary would be $(\pi / \epsilon) \sinh \left(\sqrt{|k|}\left(d(M)+2 t_{0}\right)\right) / \sqrt{|k|}$, since $\partial M=\frac{1}{\epsilon} \partial \widetilde{M}$, the boundary $\partial \widetilde{M}$ is totally geodesic in $\widetilde{M}$, and $\widetilde{M}$ has curvature bounded below by $k=k\left(K^{-}, \lambda^{ \pm}\right)$and diameter bounded above by $d(M)+2 t_{0}$. However, the optimal estimate (for general values of $K^{-}, \lambda^{ \pm}$, and $d$ ) seems to be unknown.

Proof of Theorem 1.1(iv). The upper bound on the number of boundary components follows from the Betti number theorem for Alexandrov spaces [Liu and Shen 1994] together with an easy homology argument.

As any $M \in M\left(n, K^{-}, \lambda^{ \pm}, d\right)$ is homeomorphic to its extension $\widetilde{M}$, it suffices to give a bound on the number of components of $\partial \widetilde{M}$. Consider the double $2 \widetilde{M}=$ $\widetilde{M} \bigcup_{\partial \widetilde{M}} \widetilde{M}$. This is again an Alexandrov space of curvature bounded below, since $\widetilde{M}$ has a convex boundary. The Mayer-Vietoris sequence

$$
\cdots \rightarrow H(2 \widetilde{M}) \rightarrow H(\partial \widetilde{M}) \rightarrow H(\widetilde{M}) \oplus H(\tilde{M}) \rightarrow \cdots
$$

gives, for $p=0$,

$\operatorname{rank} H_{p}(\partial M)=\operatorname{rank} H_{p+1}(2 \widetilde{M})+\operatorname{rank} H_{p}(\widetilde{M})+\operatorname{rank} H_{p}(\widetilde{M})<c\left(n, K^{-}, \lambda^{ \pm}, d\right)$, which is finite.

Explicitly, from [Liu and Shen 1994], one has the superexponential bound

$$
\sum_{p=0}^{n} \operatorname{rank} H_{p}(\tilde{M}) \leq c\left(n, K^{-}, \lambda^{ \pm}, d\right)=C_{n}^{25^{n}+n+2}(\sqrt{|k|} d+1)^{n} e^{3 n \sqrt{|k|} d}
$$

for the total Betti number, where $C_{n}=12^{n^{2}+3 n+1}$ and $k=k\left(K^{-}, K_{C}^{-}\right)$is the lower curvature bound of $\widetilde{M}$.

We remark that the number of boundary components will be at most two if there is enough combined positivity of curvature in the interior and convexity of the boundary.

We next consider precompactness of the class $\mathcal{M}\left(n, K^{-}, \lambda^{ \pm}, d\right)$.

Proof of Theorem 1.3. Take any $M \in M\left(n, K^{-}, \lambda^{ \pm}, d\right)$. Let $\widetilde{B}(x, r)$ denote a ball in an extension $\widetilde{M}$ as above, with center $x$ and radius $r$. We claim that for any $x, y \in M$ and $r>0$ that (i) $\widetilde{B}(x, r) \cap \widetilde{B}(y, r)=\varnothing$ implies $B(x, r) \cap B(y, r)=\varnothing$ and conversely (ii) $\widetilde{B}(x, r) \cap \widetilde{B}(y, r)=\varnothing$ is implied by $B(x, f(2 r)) \cap B(y, f(2 r))=\varnothing$ for some function $f$.

Part (i) is obvious, since $\widetilde{B}(x, r) \supseteq B(x, r)$ and $\widetilde{B}(y, r) \supseteq B(y, r)$. For the converse (ii), suppose $z \in \widetilde{B}(x, r) \cap \widetilde{B}(y, r)$. Then $|x z|_{\widetilde{M}},|y z|_{\widetilde{M}}<r$. Consider the 
point $z^{\prime}:=\pi(z)$, where $\pi: \widetilde{M} \rightarrow M$ is the projection map defined in (2-7). Then

$$
\begin{array}{rlrl}
\left|x z^{\prime}\right|_{\widetilde{M}} & \leq|x z|_{\widetilde{M}}+\left|z z^{\prime}\right|_{\widetilde{M}} & \\
& \leq|x z|_{\widetilde{M}}+|x z|_{\widetilde{M}} & & \text { by choice of } z^{\prime} \\
& <r+r & & \text { since }|x z|_{\widetilde{M}}<r .
\end{array}
$$

Equivalently, $z^{\prime} \in B(x, 2 r ; \widetilde{M})$.

We now assert that $z^{\prime} \in B(x, f(2 r) ; M)$ for some function $f$. (A symmetric argument with $y$ in place of $x$ will give $z^{\prime} \in B(y, f(2 r) ; M)$, so that, as desired, $B(x, f(2 r) ; M) \cap B(y, f(2 r) ; M) \neq \varnothing$.) For this, it suffices to demonstrate that $\left|x z^{\prime}\right|_{\widetilde{M}} \leq r$ implies $\left|x z^{\prime}\right|_{M} \leq f(r)$. But this is immediate from property (iii) on page 184 (as proved in Lemma 2.2), whereby we may take $f(r)=r / \epsilon$. This shows that the claim above holds.

For any metric space $X$, recall the definition of capacity (cap) from page 187 . To show that $\mathcal{M}\left(n, K^{-}, \lambda^{ \pm}, d\right)$ is precompact, we will show that $\operatorname{cap}(r, R ; \cdot)$ is bounded on the class $\mathcal{M}\left(n, K^{-}, \lambda^{ \pm}, d\right)$. It suffices to demonstrate that cap $(r, d ; \cdot)$ is so bounded for all sufficiently small $r$. Suppose $M \in M\left(n, K^{-}, \lambda^{ \pm}, d\right)$.

Set $\widetilde{d}:=d+2 t_{0}$, and recall that $d(\widetilde{M}) \leq \widetilde{d}$. In particular, $B(\widetilde{d} ; \widetilde{M})=\widetilde{M}$. Then

$\operatorname{cap}(r, d ; M)=\operatorname{cap}(r, \widetilde{d} ; M)$

$:=$ maximum \# disjoint $r$-balls $B(r ; M)$ in a $\widetilde{d}$-ball $B(\widetilde{d} ; M)$

$\leq$ maximum \# disjoint $\frac{\epsilon}{2} r$-balls $B\left(\frac{\epsilon}{2} r ; \widetilde{M}\right)$

with centers in $M$ in a $\widetilde{d}$-ball $B(\widetilde{d} ; \widetilde{M})$ by the above claim

$\leq$ maximum \# disjoint $\frac{\epsilon}{2} r$-balls $B\left(\frac{\epsilon}{2} r ; \widetilde{M}\right)$ in $\widetilde{M}$

$=: \operatorname{cap}\left(\frac{\epsilon}{2} r, \widetilde{d} ; \widetilde{M}\right)$,

and the latter is bounded by relative volume comparison in $\widetilde{M}$, in terms of only $n$, $\widetilde{K}^{-}=\widetilde{K}^{-}\left(K^{-}, \lambda^{ \pm}\right), \widetilde{\lambda}^{-}=0$, and $\widetilde{d}$, hence by only $n, K^{-}, \lambda^{ \pm}$, and $d$.

Proof of Theorem 1.4. Any $M \in \mathcal{M}\left(n, K^{-}, \lambda^{ \pm}\right.$, vol $\left.\geq v>0, d\right)$ is homeomorphic to its extension $\widetilde{M} \in \widetilde{M}\left(n, \operatorname{curv} \geq k\left(K^{-}, \lambda^{ \pm}\right)\right.$, vol $\left.\geq v, d\right)$, and the latter class contains only finitely many homeomorphism types, by the topological stability theorem of [Perelman 1992].

Proof of Theorem 1.5. Existence of the limit of the $\widetilde{M}_{i}$ 's follows (after possibly choosing a subsequence) from precompactness, since the $\widetilde{M}_{i}$ are Alexandrov spaces satisfying $d\left(\widetilde{M}_{i}\right) \leq d+2 t_{0}<\infty$.

For each $i$, one has a map $\pi_{i}: \widetilde{M}_{i} \rightarrow M_{i}$, as defined in (2-7). Lemma 2.2 and the first part of Proposition B.1 imply that $\pi_{i} \rightarrow \pi$ for some map $\pi: \lim _{\mathrm{GH}} \widetilde{M_{i}} \rightarrow$ $\lim _{\mathrm{GH}} M_{i}$. 
The inclusions $\iota_{i}: M_{i} \rightarrow \widetilde{M}_{i}$ (with Lipschitz constant equal to 1 ) subconverge to a map $\iota: \lim _{\mathrm{GH}} M_{i} \rightarrow \lim _{\mathrm{GH}} \widetilde{M}_{i}$, again by the first part of Proposition B.1.

One can write any point $x \in C_{M_{i}}=\partial M_{i} \times_{\phi}\left[0, t_{0}\right]$ in coordinates as $x=\left(x^{\prime}, r\right)$, where $x^{\prime} \in \partial M_{i}$ and $r \in\left[0, t_{0}\right]$.

Define a map $H_{i, t}: \widetilde{M}_{i} \rightarrow \widetilde{M}_{i}$, for any time $0 \leq t \leq 1$, by

$$
H_{i, t}(x)= \begin{cases}x & \text { if } x \in M_{i}, \\ \left(x^{\prime}, t r\right) & \text { if } x=\left(x^{\prime}, r\right) \in C_{M_{i}} .\end{cases}
$$

Then $H_{i, 0}(x)=\iota_{i} \circ \pi_{i}(x)$ and $H_{i, 1}(x)=\operatorname{id} \widetilde{M_{i}}(x)=x$.

Let $H_{i}(x, t)=H_{i, t}(x)$. For any $x, y \in \widetilde{M}_{i}$ and times $0 \leq s, t \leq 1$, we will in a moment show

$$
\left|H_{i}(x, t) H_{i}(y, s)\right|_{\widetilde{M}_{i}} \leq L|(x, t)(y, s)|_{\widetilde{M}_{i} \times I}
$$

for some constant $L=L\left(\epsilon, t_{0}\right)<\infty$. Here, the metric on the space-time $\widetilde{M}_{i} \times I$ is taken to be the direct product metric.

Given inequality (2-14), the proof of Theorem 1.5 can be finished as follows. By Proposition B.2, the homotopies $H_{i, t}$ from $\iota_{i} \circ \pi_{i}$ to $\mathrm{id}_{\widetilde{M}_{i}}$ give rise to a limit homotopy $\iota \circ \pi \simeq \mathrm{id}_{\lim _{\mathrm{GH}} \widetilde{M}_{i}}$. On the other hand, for all $i, \pi_{i} \circ \iota_{i}=\operatorname{id}_{M_{i}}$ on $M_{i}$, so $\pi \circ \iota=\operatorname{id}_{\lim _{\mathrm{GH}} M_{i}}$. Therefore $\lim _{\mathrm{GH}} \widetilde{M}_{i} \simeq \lim _{\mathrm{GH}} M_{i}$.

It only remains to prove inequality (2-14). To accomplish this, there are only three cases to consider:

Case: $x, y \in M_{i}$.

$$
\begin{array}{rlrl}
\left|H_{i}(x, t) H_{i}(y, s)\right|_{\widetilde{M}_{i}} & =|x y| \widetilde{M}_{i} & & \text { by definition of } H_{i} \\
& \leq|(x, t)(y, s)|_{\widetilde{M}_{i} \times I} & \text { for any } s, t \in I .
\end{array}
$$

Case: $x, y \in C_{M_{i}}$. This case requires a preparatory inequality, Lemma 2.3 below, whose proof is given shortly after we otherwise finish proving Theorem 1.5.

Write $x=\left(x^{\prime}, u\right)$ and $y=\left(y^{\prime}, v\right)$. Then Lemma 2.3 shows that

$$
\left|\left(x^{\prime}, t u\right)\left(y^{\prime}, t u\right)\right|_{\widetilde{M}_{i}} \leq \frac{1}{\epsilon}\left|\left(x^{\prime}, u\right)\left(y^{\prime}, u\right)\right|_{\widetilde{M}_{i}}
$$

Proceeding, observe that

$$
|u v|_{I} \leq\left|\left(x^{\prime}, u\right)\left(y^{\prime}, v\right)\right|_{\widetilde{M}_{i}}=|x y|{\widetilde{M_{i}}}
$$

We will also use the algebraic inequality that

$$
|t u-s v| \leq \sqrt{2} \max \left\{1, t_{0}\right\} \cdot\left(|u-v|^{2}+|t-s|^{2}\right)^{1 / 2}
$$


whenever $0 \leq t \leq 1$ and $0 \leq v \leq t_{0}$. To see this, write $t u-s v=t(u-v)+v(t-s)$, so that

$$
\begin{aligned}
|t u-s v| & \leq \max \{t\}|u-v|+\max \{v\}|t-s| \\
& \leq \max \{\max t, \max v\}(|u-v|+|t-s|) \\
& \leq \max \{\max t, \max v\} \sqrt{2}\left(|u-v|^{2}+|t-s|^{2}\right)^{1 / 2} .
\end{aligned}
$$

\section{Hence}

$$
\begin{aligned}
& \left|H_{i}(x, t) H_{i}(y, s)\right|_{\widetilde{M}_{i}}=\left|\left(x^{\prime}, t u\right)\left(y^{\prime}, s v\right)\right|{\widetilde{M_{i}}} \\
& \text { by definition of } H_{i} \\
& \leq\left|\left(x^{\prime}, t u\right)\left(y^{\prime}, t u\right)\right|_{\widetilde{M}_{i}}+\left|\left(y^{\prime}, t u\right)\left(y^{\prime}, s v\right)\right|_{\widetilde{M}_{i}} \\
& \leq \frac{1}{\epsilon}\left|\left(x^{\prime}, u\right)\left(y^{\prime}, u\right)\right|_{\widetilde{M}_{i}}+\left|\left(y^{\prime}, t u\right)\left(y^{\prime}, s v\right)\right|_{\widetilde{M}_{i}} \\
& =\frac{1}{\epsilon}\left|\left(x^{\prime}, u\right)\left(y^{\prime}, u\right)\right|_{\widetilde{m}_{i}}+|t u-s v|_{I} \\
& \leq \frac{1}{\epsilon}\left|\left(x^{\prime}, u\right)\left(y^{\prime}, u\right)\right|_{\widetilde{M}_{i}}+\max \left\{1, t_{0}\right\} \sqrt{2}\left(|u v|_{I}^{2}+|t s|_{I}^{2}\right)^{1 / 2} \text { by estimate (2-18) above } \\
& \leq \frac{1}{\epsilon}\left(\left|\left(x^{\prime}, u\right)\left(y^{\prime}, v\right)\right|_{\widetilde{M}_{i}}+\left|\left(y^{\prime}, v\right)\left(y^{\prime}, u\right)\right|_{\widetilde{M}_{i}}\right)+\max \left\{1, t_{0}\right\} \sqrt{2}\left(|u v|_{I}^{2}+|t s|_{I}^{2}\right)^{1 / 2} \\
& \text { by the triangle inequality } \\
& =\frac{1}{\epsilon}\left(|x y|_{\widetilde{M}_{i}}+|u v|_{I}\right)+\max \left\{1, t_{0}\right\} \sqrt{2}\left(|u v|_{I}^{2}+|t s|_{I}^{2}\right)^{1 / 2} \\
& \leq \frac{1}{\epsilon}\left(|x y|_{\widetilde{M}_{i}}+|x y|_{\widetilde{M}_{i}}\right)+\max \left\{1, t_{0}\right\} \sqrt{2}\left(|x y|_{\widetilde{M}_{i}}^{2}+|t s|_{I}^{2}\right)^{1 / 2}
\end{aligned}
$$$$
\text { by the observation (2-17) above }
$$$$
=\frac{2}{\epsilon}|x y|_{\widetilde{M}_{i}}+\max \left\{1, t_{0}\right\} \sqrt{2}|(x, t)(y, s)|_{\widetilde{M}_{i} \times I}
$$$$
\leq \frac{2}{\epsilon}|(x, t)(y, s)|_{\widetilde{M}_{i} \times I}+\max \left\{1, t_{0}\right\} \sqrt{2}|(x, t)(y, s)|_{\widetilde{M}_{i} \times I}
$$$$
=\left(\frac{2}{\epsilon}+\max \left\{1, t_{0}\right\} \sqrt{2}\right)|(x, t)(y, s)|_{\widetilde{M}_{i} \times I}
$$

Case: $x \in M_{i}, y \in C_{M_{i}}$. Choose $z \in \partial M_{i}$ and $r \in I$ such that $(z, r)$ belongs to a minimal geodesic $[(x, t)(y, s)]_{\widetilde{M}_{i} \times I}$. This is possible since $[x y]_{\widetilde{M}_{i}}$ must cross $\partial M_{i}$, which implies that $[(x, t)(y, s)]_{\widetilde{M}_{i} \times I}$ must cross the subset $\partial M_{i} \times I \subset \widetilde{M}_{i} \times I$.

$$
\left|H_{i}(x, t) H_{i}(y, s)\right|_{\widetilde{M}_{i}} \leq\left|H_{i}(x, t) H_{i}(z, r)\right|_{\widetilde{M}_{i}}+\left|H_{i}(z, r) H_{i}(y, s)\right| \widetilde{M}_{i}
$$

by the triangle inequality

$$
\leq|(x, t)(z, r)|_{\widetilde{M}_{i} \times I}+\left|H_{i}(z, r) H_{i}(y, s)\right|_{\widetilde{M}_{i}}
$$

by (2-15) of the first case, since $x, z \in M_{i}$,

$$
\leq|(x, t)(z, r)|_{\widetilde{M}_{i} \times I}+\left(\frac{2}{\epsilon}+\max \left\{1, t_{0}\right\} \sqrt{2}\right)|(z, r)(y, s)|_{\widetilde{M}_{i} \times I}
$$

by the previous case's result, since $z, y \in C_{M_{i}}$,

$$
\leq \max \left\{1,\left(\frac{2}{\epsilon}+\max \left\{1, t_{0}\right\} \sqrt{2}\right)\right\}\left(|(x, t)(z, r)|_{\widetilde{M}_{i} \times I}+|(z, r)(y, s)|_{\widetilde{M}_{i} \times I}\right)
$$

$$
=\left(\frac{2}{\epsilon}+\max \left\{1, t_{0}\right\} \sqrt{2}\right)|(x, t)(y, s)|_{\widetilde{M}_{i} \times I}
$$

by choice of $z$ and $r$. 
Considering the results of these three cases together, this proves inequality (2-14) and hence Theorem 1.5.

Lemma 2.3. In the setting as above, $\left|\left(x^{\prime}, t u\right)\left(y^{\prime}, t u\right)\right|_{\widetilde{M}_{i}} \leq \frac{1}{\epsilon}\left|\left(x^{\prime}, u\right)\left(y^{\prime}, u\right)\right|_{\widetilde{M}_{i}}$.

Proof. We can prove this using a segment subdivision method virtually identical to that in Lemma 2.2.

Analogously to the projection-type map defined in (2-7), define a map

$$
\pi_{t u}: \widetilde{M}_{i} \rightarrow M_{i} \cup\left(\partial M_{i} \times_{\phi}[0, t u]\right)
$$

by

$$
\pi_{t u}(w)=\left\{\begin{array}{l}
w \text { if } w \in M_{i} \cup\left(\partial M_{i} \times_{\phi}[0, t u]\right), \\
\text { orthogonal projection of } w \text { onto } \partial M_{i} \times_{\phi}\{t u\} \text { if } w \in \partial M_{i} \times_{\phi}\left[t u, t_{0}\right] .
\end{array}\right.
$$

(So in particular, $\pi_{0}$ is just the map $\pi$ from Lemma 2.2.)

Now the proof may be repeated verbatim from Lemma 2.2, if one substitutes $M_{i} \cup\left(\partial M_{i} \times_{\phi}[0, t u]\right)$ for $M_{i}, \quad \partial M_{i} \times_{\phi}\left[t u, t_{0}\right]$ for $C_{M_{i}}, \quad$ and $\pi_{t u}$ for $\pi$.

Now we generalize several of the results above to Ricci curvature.

Proof of Theorem 1.9. (i) Fix a constant $t_{0}$ as in Lemma 1.7. For any $M \in$ $M\left(n, r^{-}, \lambda^{ \pm}, d\right)$, consider its smoothed extension $\widetilde{M_{\delta}}$, which results from applying the Ricci version of Lemma 1.7 together with Theorem 1.8. We may assume that $\delta$ has been chosen so that $\delta<t_{0}$. We will restrict $\delta$ further in a moment. Let $r_{1}^{-}:=\min \left\{r^{-}, r_{C_{M}}^{-}\right\}$be the minimum of the lower Ricci curvature bound of $M$ and the lower Ricci curvature bound $r_{C_{M}}^{-}=r_{C_{M}}^{-}\left(r^{-}, \lambda^{ \pm}\right)$of $C_{M}$. Then ${ }^{2} \operatorname{ric}\left(\widetilde{M_{\delta}}\right) \geq r_{1}^{-}$.

Since the smoothing on the $\delta$-neighborhood of $\partial M$ is effected through linear interpolations of the metrics of $M$ and $C_{M}$ written in the form $g=d t^{2}+g_{t}$, where $t \in(-\delta, \delta)$ denotes the signed distance to $\partial M$, and the metric of $M \bigcup_{\partial M} C_{M}$ is unchanged off the $\delta$-neighborhood of $\partial M$, it follows that

$$
\widetilde{M}_{\delta} \stackrel{\mathrm{GH}}{\longrightarrow} M \bigcup_{\partial M} C_{M} \quad \text { as } \delta \rightarrow 0 .
$$

Thus

$$
d\left(\widetilde{M_{\delta}}\right) \leq d\left(M \bigcup_{\partial M} C_{M}\right)+\tau(\delta) \leq d+2 t_{0}+\tau(\delta),
$$

where $\tau$ is a function satisfying $\tau(\delta) \rightarrow 0$ as $\delta \rightarrow 0$. We can assume $\delta$ is chosen sufficiently small so that $\tau(\delta) \leq 1$, say.

${ }^{2}$ Technically, Theorem 1.8 was stated only for strict inequality of the Ricci curvature tensor, but if $\operatorname{ric}(M) \geq r^{-}$and $\operatorname{ric}\left(C_{M}\right) \geq r_{C_{M}}$ then $\operatorname{ric}(M)>r^{-}-\eta$ and $\operatorname{ric}\left(C_{M}\right)>r_{C_{M}}-\eta$ for all $\eta>0$. So, provided that $\delta$ is sufficiently small relative to $M$ and $C_{M}, \operatorname{ric}\left(\widetilde{M_{\delta}}\right)>\min \left\{r^{-}-\eta, r_{C_{M}}-\eta\right\}$ for all $\eta>0$ by Theorem 1.8, which implies $\operatorname{ric}\left(\widetilde{M_{\delta}}\right) \geq r_{1}^{-}$. 
Since $\widetilde{M_{\delta}}$ has totally geodesic boundary by construction, absolute volume comparison applies to $\widetilde{M_{\delta}}$, and

$$
\operatorname{vol}\left(\widetilde{M_{\delta}}\right) \leq V\left(n, r_{1}^{-}, d+2 t_{0}+1\right)=V\left(n, r^{-}, \lambda^{ \pm}, d\right) .
$$

Since $M$ is not isometrically embedded in $\widetilde{M_{\delta}}$, it cannot be asserted directly that $\operatorname{vol}(M) \leq \operatorname{vol}\left(\widetilde{M_{\delta}}\right)$. However, (2-19) implies $\operatorname{vol}\left(\widetilde{M}_{\delta}\right) \rightarrow \operatorname{vol}\left(M \bigcup_{\partial M} C_{M}\right)$ by volume continuity [Cheeger and Colding 1997, Theorem 5.9]. Since $M$ is isometrically embedded in $M \bigcup_{\partial M} C_{M}$, it follows that

$$
\operatorname{vol}(M) \leq \operatorname{vol}\left(M \bigcup_{\partial M} C_{M}\right) \leq \operatorname{vol}\left(\widetilde{M_{\delta}}\right)+\tau(\delta) \leq V\left(n, r^{-}, \lambda^{ \pm}, d\right)
$$

for some upper bound $V\left(n, r^{-}, \lambda^{ \pm}, d\right)$.

(ii) The proof is similar to that of Theorem 1.1(iv), except that here instead of the Betti number for Alexandrov spaces, we can use that closed $n$-dimensional manifolds having lower Ricci curvature bound and upper diameter bound have bounded first Betti number [Gromov 1999, 5.20, 5.21].

(iii) The proof is exactly the same as in Theorem 1.1(iii), with $\widetilde{M_{\delta}}$ in place of $\widetilde{M}$ and $i_{\partial}(\widetilde{M}) \geq t_{0}$ replaced by $i_{\partial}\left(\widetilde{M_{\delta}}\right) \geq t_{0}-\delta$.

(iv) The proof is the same as in Theorem 1.3, although the very last line there, which invoked relative volume comparison in $\widetilde{M}$, needs here further justification. Given that $M$ and $C_{M}$ only have a lower Ricci curvature bound, it is still true that relative volume comparison holds for the glued space $\widetilde{M}=M \bigcup_{\partial M} C_{M}$, by [Cheeger and Colding 1997, Theorem 5.9], since the convergence (2-19) of $\widetilde{M}_{\delta}$ to $\widetilde{M}$ is noncollapsing in the sense that $\operatorname{vol}\left(B\left(x, 1 ; \widetilde{M_{\delta}}\right)\right) \geq v>0$ for some $v$ and all $x$ and $\delta>0$. The model space for the relative volume comparison here is the simply-connected $n$-dimensional space of constant curvature $r_{1}^{-}$.

2.3. Questions. It would be interesting to see if the upper bound $\lambda^{+}$could be eliminated from Theorems 1.1-1.5 and Theorem 1.9. The only place where this bound was used was in invoking the Gauss equations to produce a lower curvature bound for the extension.

It also remains to be seen for which of the results can the lower bound $\lambda^{-}$on the second fundamental form be replaced by a lower bound on the mean curvature, or perhaps by a bound on integral of mean curvature.

In this direction of considering weaker curvature bounds, we remark that a precompactness and convergence theorem has recently been proved in [Anderson et al. 2004], in the noncollapsing regime for which one has all of the injectivity radius bounds for $i_{\text {int }}(M)$, inj $(\partial M)$, and $i_{\partial}(M)$. They considered the class $\mathcal{M}\left(n, r_{0}, i_{0}, H_{0}, d\right)$, consisting of manifolds with boundary $(M, \partial M)$ such that $\operatorname{dim} M=n,\|\operatorname{ric}(M)\|_{L^{\infty}(M)} \leq r_{0},\|\operatorname{ric}(\partial M)\|_{L^{\infty}(\partial M)} \leq r_{0}, i_{\text {int }}(M) \geq i_{0}$, 
$\operatorname{inj}(\partial M) \geq i_{0}, i_{\partial} \geq i_{0},\|H\|_{\operatorname{Lip}(\partial M)} \leq H_{0}$, and $d(M) \leq d$, and showed that it is precompact in the $C^{r}$ topology for each $r<2$. The main idea of their proof involved boundary harmonic coordinates and boundary harmonic radius.

Theorem 1.4 suggests that there is an underlying stability accounting for the finiteness. Namely, if $N \in \mathcal{M}=\mathcal{M}\left(n, K^{-}, \lambda^{ \pm}\right.$, vol $\left.>v>0, d\right)$ then does there exist an $\epsilon>0$ such that any $M \in \mathcal{M}$ with $d_{\mathrm{GH}}(M, N)<\epsilon$ is homeomorphic to $N$ ? This could be answered if it could be shown that Gromov-Hausdorff closeness of two manifolds implied their (Alexandrov) extensions were correspondingly close.

\section{Appendix A. Injectivity radii: definitions}

In a manifold with boundary, the usual Riemannian exponential map is not well defined because geodesics may bifurcate. Here we give two definitions of injectivity radii which are used in this paper.

Definition. For a Riemannian manifold with boundary $(M, \partial M)$, and $p \in M$, define $i_{\text {int }}(p)$ to be the supremum over all $r>0$ such that any unit-speed geodesic $\gamma:\left[0, t_{\gamma}\right] \rightarrow M$ issuing from $p$ is distance minimizing up to the distance $\min \left(t_{\gamma}, r\right)$, where $t_{\gamma}$ is the first time $\gamma$ intersects $\partial M$ (so $t_{\gamma}=\infty$ if $\gamma \cap \partial M=\varnothing$ ). Define $i_{\text {int }}(M)=\inf _{p \in M}\left\{i_{\text {int }}(p)\right\}$, the interior injectivity radius.

For a closed manifold $M$, or a manifold with locally convex boundary, $i_{\text {int }}(M)$ coincides with the usual notion of injectivity radius, often denoted $\operatorname{inj}(M)$, defined via the exponential map.

Definition. For a Riemannian manifold with boundary $(M, \partial M)$, and $p \in \partial M$, define $i_{\partial}(p)$ to be the supremum over all $r>0$ such that any minimizing geodesic $\gamma$ issuing from $p$ normally to $\partial M$ uniquely minimizes distance to $\partial M$ up to distance $r$ (that is, $\gamma(0)=p$ and $d(\gamma(r), \partial M)=r)$. Define $i_{\partial}(M)=\inf _{p \in \partial M}\left\{i_{\partial}(p)\right\}$, the boundary injectivity radius of $(M, \partial M)$.

So, if $i_{\partial}(M) \geq i_{0}$, then $M$ admits an inward tubular neighborhood of radius at least $i_{0}$. One has the Klingenberg-type estimate $i_{\partial}(M)=\min \{\operatorname{Foc}(\partial M), L / 2\}$, where $\operatorname{Foc}(\partial M)$ is the minimum focal distance for the normal exponential map of the boundary and $L$ represents the shortest length of a segment meeting $\partial M$ at right angles at both its endpoints. It is known that

$$
\operatorname{Foc}(\partial M) \geq \frac{1}{\sqrt{K^{+}}} \arctan \left(\frac{\sqrt{K^{+}}}{\lambda^{+}}\right) \quad \text { if } K_{M} \leq K^{+} \text {and } I I_{\partial M} \leq \lambda^{+} .
$$

An example of these definitions is $M=\left(\mathbb{R}^{n} \backslash B^{n}(r), g_{\text {std }}\right)$, the Euclidean space with a ball of radius $r$ removed. It has $i_{\text {int }}(M)=\infty$ and $i_{\partial}(M)=\infty$. 
Another is $M=\left(S^{n}(1) \backslash B^{n}(r), g_{\text {std }}\right)$, the standard sphere of radius 1 with a ball of radius $r$ removed, which, for $0<r<\pi$, has

$$
i_{\text {int }}(M)=\left\{\begin{array}{ll}
\pi & \text { for } r \leq \pi / 2, \\
\infty & \text { for } r>\pi / 2,
\end{array} \quad \text { and } \quad i_{\partial}(M)=\pi-r .\right.
$$

\section{Appendix B. Gromov-Hausdorff convergence}

This section gives background on Gromov-Hausdorff convergence. In particular, it details two functorial properties of maps: one for surjective, Lipschitz maps and another for Lipschitz homotopy equivalences. These are used for the proofs of Corollary 1.2 and Theorem 1.5. Additional background on Gromov-Hausdorff convergence may be found in [Fukaya 1990; Petersen 1993].

Let $Z$ be a metric space. The Hausdorff distance $d_{\mathrm{H}}^{Z}(X, Y)$ between two subsets $X, Y \subseteq Z$ is defined to be $d_{\mathrm{H}}^{Z}(X, Y):=\inf \{\epsilon>0: B(X, \epsilon) \supseteq Y, B(Y, \epsilon) \supseteq X\}$, where $B(X, \epsilon)=\{z \in Z: d(z, X)<\epsilon\}$ denotes the metric $Z$-ball about $X$ of radius $\epsilon$.

Definition. The Gromov-Hausdorff distance between two metric spaces $X$ and $Y$ is $d_{\mathrm{GH}}(X, Y)=\inf \left\{d_{\mathrm{H}}^{Z}\left(i_{X}(X), i_{Y}(Y)\right)\right\}$, where the infimum is taken over all metric spaces $Z$ and all distance-preserving embeddings $i_{X}: X \hookrightarrow Z$ and $i_{Y}: Y \hookrightarrow Z$.

We say a sequence of metric spaces $X_{i}$ converges to $X$, and write $X_{i} \stackrel{\mathrm{GH}}{\longrightarrow} X$, if $d_{\mathrm{GH}}\left(X, X_{i}\right) \rightarrow 0$ as $i \rightarrow \infty$. In practice one usually uses the following formulation to verify that a convergence occurs.

Definition. An $\epsilon$-Hausdorff approximation $\psi: X \rightarrow Y$ is a (not necessarily continuous) map such that $\psi(X)$ is an $\epsilon$-net in $Y$, that is, $B(\psi(X), \epsilon ; Y)=Y$, and $\psi$ is an $\epsilon$-almost isometry, that is, $\left|d_{Y}\left(\psi\left(x_{1}\right), \psi\left(x_{2}\right)\right)-d_{X}\left(x_{1}, x_{2}\right)\right| \leq \epsilon$ for all $x_{1}, x_{2} \in X$.

One has the notion of convergence of points.

Definition. If $X_{i} \stackrel{\mathrm{GH}}{\longrightarrow} X$ via $\epsilon_{i}$-Hausdorff approximations $\psi_{i}: X_{i} \rightarrow X$, we say points $x_{i} \in X_{i}$ converge to a point $x \in X$ (and write $x_{i} \mapsto x$ ) if $d\left(\psi_{i}\left(x_{i}\right), x\right) \rightarrow 0$.

This permits one to define convergence of maps.

Definition. If $f_{i}: X_{i} \longrightarrow Y_{i}$ are maps, $X_{i} \stackrel{\mathrm{GH}}{\longrightarrow} X$ and $Y_{i} \stackrel{\mathrm{GH}}{\longrightarrow} Y$, then the $f_{i}$ converge to a map $f: X \rightarrow Y$ if $f_{i}\left(x_{i}\right) \mapsto f(x)$ whenever $X_{i} \ni x_{i} \mapsto x \in X$; for this we write $f_{i} \rightarrow f$.

The following proposition is a slight modification of [Gromov 1999, Section $3.11 \frac{1}{2}{ }_{+}$, exercise (c) on p. 78].

Proposition B.1. Let $X_{i}$ and $Y_{i}$ be metric spaces with $X_{i} \stackrel{\mathrm{GH}}{\longrightarrow} X$ and $Y_{i} \stackrel{\mathrm{GH}}{\longrightarrow} Y$, with $X$ and $Y$ compact. Suppose that for all $i$ there exist L-Lipschitz maps $f_{i}: Y_{i} \rightarrow X_{i}$. Then there exists an L-Lipschitz map $f: Y \rightarrow X$. If the $f_{i}$ are also surjective, then so is the limit map $f$. 
For the proof, see [Wong 2006].

Proposition B.2. Suppose $X_{j} \stackrel{\mathrm{GH}}{\longrightarrow} X$ and $Y_{j} \stackrel{\mathrm{GH}}{\longrightarrow} Y$ for complete metric spaces $X_{j}$ and $Y_{j}$, with $X$ and $Y$ compact. Suppose that for each $j$ there exist continuous maps $f_{j}: X_{j} \rightarrow Y_{j}, g_{j}: Y_{j} \rightarrow X_{j}, f: X \rightarrow Y$, and $g: Y \rightarrow X$ such that $f_{j} \rightarrow f$ and $g_{j} \rightarrow g$.

Suppose that for each $j$, there exist maps $H_{j}: X_{j} \times I \rightarrow X_{j}$ such that

$$
H_{j}(x, 0)=g_{j} \circ f_{j}(x), \quad H_{j}(x, 1)=\operatorname{id}_{X_{j}}(x)=x,
$$

and $H_{j}(x, t)$ is globally Lipschitz in $x, t$, uniformly in $j$ (where $X_{j} \times I$ is equipped with the direct product metric). Suppose also there exist maps $\bar{H}_{j}: Y_{j} \times I \rightarrow Y_{j}$ for each $j$ such that

$$
\bar{H}_{j}(x, 0)=f_{j} \circ g_{j}(x), \quad \bar{H}_{j}(x, 1)=\operatorname{id}_{Y_{j}}(x)=x,
$$

and $\bar{H}_{j}(x, t)$ is globally Lipschitz in $x, t$, uniformly in $j$ (where $Y_{j} \times I$ equipped with the direct product metric). Then $X$ and $Y$ are homotopy equivalent (via a Lipschitz homotopy equivalence).

The theorem is illustrated by this diagram:

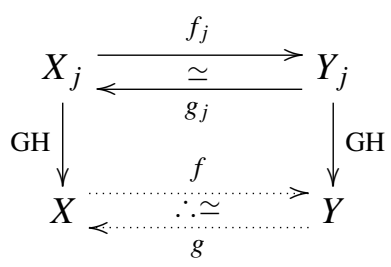

Proof. By assumption, there exists $L<\infty$ such that

$$
\left|H_{j}(x, t) H_{j}(y, s)\right|_{X_{j}} \leq L|(x, t)(y, s)|_{X_{j} \times I}
$$

for all $x, y \in X_{j}$ and all $0 \leq s, t \leq 1$; in particular, the $H_{j}$ are equicontinuous. Since $X$ is compact and hence bounded, $d\left(X_{j}\right) \leq d<\infty$ for all sufficiently large $j$, whence the $H_{j}$ are also uniformly bounded.

Note that $X_{j} \times I \stackrel{\mathrm{GH}}{\longrightarrow} X \times I$ since $X_{j} \stackrel{\mathrm{GH}}{\longrightarrow} X$.

By Arzelà-Ascoli, $H_{j}$ subconverge to a map $H: X \times I \rightarrow X$ satisfying

$$
|H(x, t) H(y, s)|_{X} \leq L|(x, t)(y, s)|_{X \times I}
$$

for all $x, y \in X$ and all $0 \leq s, t \leq 1$, by the first part of Proposition B.1. In particular, $H$ is jointly continuous with respect to $x, t$. Also, since $g_{j} \circ f_{j} \rightarrow g \circ f$ and $\operatorname{id}_{X_{j}} \rightarrow \operatorname{id}_{X}$, we have $H(x, 0)=g \circ f(x)$ and $H(x, 1)=\operatorname{id}_{X}(x)=x$. Therefore $H$ is a homotopy from $g \circ f$ to $\operatorname{id}_{X}$, that is, $g \circ f \simeq \operatorname{id}_{X}$.

A symmetric argument, with $\bar{H}_{j}$ in place of $H_{j}$, yields a homotopy $\bar{H}$ between $f \circ g$ and $\operatorname{id}_{Y}$, that is, $f \circ g \simeq \operatorname{id}_{Y}$. Therefore $X \simeq Y$. 
If the limiting homotopies are not uniformly (in $j$ ) Lipschitz, the limit spaces need not be homotopy equivalent. For example, take $X_{j}$ (for all $j$ ) identically to be a given point $\{x\}$ in the unit sphere $S^{2}(1)$, and let $Y_{j}:=S^{2}(1) \backslash B^{2}(-x, 1 / j)$, where $-x$ denotes the antipode to $x$. Let $\bar{H}_{j}$ be the obvious deformation retraction of $Y_{j}$ to $X_{j}$. Although $Y_{j} \simeq X_{j}$ for each $j$, the limit space $Y=S^{2}(1)$ is not homotopy equivalent to $X=\{x\}$.

\section{Acknowledgment}

The author thanks Professor Stephanie Alexander for helpful discussions and suggestions.

\section{References}

[Aleksandrov and Zalgaller 1967] A. D. Aleksandrov and V. A. Zalgaller, Intrinsic geometry of surfaces, Translations of Mathematical Monographs 15, American Mathematical Society, Providence, R.I., 1967. MR 35 \#7267 Zbl 0146.44103

[Alexander et al. $\geq 2008$ ] S. B. Alexander, M. Ghomi, and J. Wong, "Topology of nonnegatively curved hypersurfaces spanning a prescribed boundary in $\mathbb{R}^{n}$ ", preprint, Available at http://www. math.uiuc.edu/ sba/fill.pdf.

[Alexandrov 1948] Александров, А. Д., Внутренняя Геометрия Выпуклых Поверхностей, OGIZ, Moscow-Leningrad, 1948. MR 10,619c

[Anderson et al. 2004] M. Anderson, A. Katsuda, Y. Kurylev, M. Lassas, and M. Taylor, "Boundary regularity for the Ricci equation, geometric convergence, and Gel'fand's inverse boundary problem”, Invent. Math. 158:2 (2004), 261-321. MR 2005h:53051 Zbl 02132012

[Bartnik 1993] R. Bartnik, "Quasi-spherical metrics and prescribed scalar curvature”, J. Diff. Geom. 37:1 (1993), 31-71. MR 93i:53041 Zbl 0786.53019

[Bartnik 1997] R. Bartnik, "Energy in general relativity", pp. 5-27 in Tsing Hua lectures on geometry \& analysis (Hsinchu, 1990-1991), edited by S.-T. Yau, Internat. Press, Cambridge, MA, 1997. MR 99a:83028 Zbl 0884.53065

[Burago et al. 2001] D. Burago, Y. Burago, S. Ivanov, A course in metric geometry, Graduate Studies in Mathematics 33, American Mathematical Society, Providence, RI, 2001. MR 2002e:53053 Zbl 0981.51016

[Cheeger and Colding 1997] J. Cheeger and T. H. Colding, "On the structure of spaces with Ricci curvature bounded below. I”, J. Diff. Geom. 46:3 (1997), 406-480. MR 98k:53044 Zbl 0902.53034

[Fukaya 1990] K. Fukaya, "Hausdorff convergence of Riemannian manifolds and its applications", pp. 143-238 in Recent topics in differential and analytic geometry, edited by T. Ochiai, Adv. Stud. Pure Math. 18, Academic Press, Boston, 1990. MR 92k:53076 Zbl 0754.53004

[Gromov 1999] Misha Gromov, Metric structures for Riemannian and non-Riemannian spaces, Progress in Mathematics 152, Birkhäuser, Boston, 1999. MR 2000d:53065 Zbl 0953.53002

[Kim et al. 2005] H. Kim, S. Oh, and J.-w. Yim, "Smooth surface extension with curvature bound", Comput. Aided Geom. Design 22:1 (2005), 27-43. MR 2108403 Zbl 1085.65019

[Kodani 1990] S. Kodani, "Convergence theorem for Riemannian manifolds with boundary", Compositio Math. 75:2 (1990), 171-192. MR 92b:53066 Zbl 0703.53043 
[Kosovskiı̌ 2002] N. N. Kosovskiı̆, "Gluing of Riemannian manifolds of curvature $\geq \kappa$ ", Algebra $i$ Analiz 14:3 (2002), 140-157. MR 2003d:53053 Zbl 1047.53019

[Kronwith 1979] S. Kronwith, "Convex manifolds of nonnegative curvature", J. Diff. Geom. 14:4 (1979), 621-628. MR 82k:53063 Zbl 0473.53050

[Lewy 1951] H. Lewy, "On the boundary behavior of minimal surfaces", Proc. Nat. Acad. Sci. U. S. A. 37 (1951), 103-110. MR 14,168b Zbl 0042.15702

[Liu and Shen 1994] Z.-d. Liu and Z. M. Shen, "On the Betti numbers of Alexandrov spaces", Ann. Global Anal. Geom. 12:2 (1994), 123-133. MR 95b:53055 Zbl 0824.53041

[Miao 2002] P. Miao, "Positive mass theorem on manifolds admitting corners along a hypersurface", Adv. Theor. Math. Phys. 6:6 (2002), 1163-1182. MR 2005a:53065

[Nitsche 1973] J. C. C. Nitsche, "A new uniqueness theorem for minimal surfaces", Arch. Rational Mech. Anal. 52 (1973), 319-329. MR 49 \#6008 Zbl 0285.49001

[Perelman 1992] G. Perelman, “A. D. Alexandrov's spaces with curvature bounded from below, II”, preprint, 1992, Available at http://www.math.psu.edu/petrunin/papers/perelmanASWCBFB2+.pdf.

[Perelman 1997] G. Perelman, "Construction of manifolds of positive Ricci curvature with big volume and large Betti numbers", pp. 157-163 in Comparison geometry (Berkeley, CA, 199394), edited by K. Grove and P. Petersen, Math. Sci. Res. Inst. Publ. 30, Cambridge Univ. Press, Cambridge, 1997. MR 98h:53062 Zbl 0890.53038

[Petersen 1993] P. Petersen, V, “Gromov-Hausdorff convergence of metric spaces", pp. 489-504 in Differential geometry: Riemannian geometry (Los Angeles, CA, 1990), edited by R. Greene, Proc. Sympos. Pure Math. 54, Amer. Math. Soc., Providence, RI, 1993. MR 94b:53079 Zbl 0788.53037

[Shi and Tam 2004] Y. Shi and L.-F. Tam, "Quasi-spherical metrics and applications", Comm. Math. Phys. 250:1 (2004), 65-80. MR 2005m:53056 Zbl 1074.53033

[Shikata 1966] Y. Shikata, "On a distance function on the set of differentiable structures", Osaka J. Math. 3 (1966), 65-79. MR 34 \#2022 Zbl 0168.44301

[Wang 1997] H.-H. Wang, Boundary convexity on manifolds with nonnegative Ricci curvature, $\mathrm{PhD}$ thesis, Indiana University, 1997.

[Wei 1989] G. Wei, Aspects of positively Ricci curved spaces: new examples and the fundamental group, PhD thesis, Stony Brook University, 1989.

[Wong 2006] J. Wong, Collapsing manifolds with boundary, $\mathrm{PhD}$ thesis, University of Illinois at Urbana-Champaign, 2006.

[Wong 2007] J. Wong, "Collapsing manifolds with boundary", preprint, 2007. arXiv 0711.3483v1

Received May 2, 2007.

JEREMY WONG

DEPARTMENT OF MATHEMATICS

UNIVERSITY OF TORONTO

TORONTO, ON M5S 2E4

CANADA

jawong1@math.toronto.edu 